\title{
Brillouin Scattering Study of Propylene Carbonate: An Evaluation of Phenomenological and Mode Coupling Analyses
}

\author{
Alexander Brodin ${ }^{1}$, Martin Frank ${ }^{1,2}$, Sabine Wiebel ${ }^{2}$, Guoqing Shen ${ }^{1 *}$, Joachim Wuttke ${ }^{2}$, and H.Z. Cummins ${ }^{1}$ \\ 1 Physics Department, City College of the City University of New York, New York, NY 10031 \\ 2 Physics Department E13, The Technical University of Munich, D-85747 Garching, GERMANY
}

(November 2, 2018)

\begin{abstract}
Brillouin scattering spectra of the molecular glassformer propylene carbonate (PC) in the temperature range $140 \mathrm{~K}$ to $350 \mathrm{~K}$ were analyzed using both the phenomenological Cole-Davidson memory function and a hybrid memory function consisting of the Cole-Davidson function plus a power-law term representing the critical decay part of the fast $\beta$ relaxation. The spectra were also analyzed using the extended two-correlator schematic MCT model recently employed by Götze and Voigtmann to analyze depolarized light backscattering, dielectric, and neutron-scattering spectra of PC [W. Götze and Th. Voigtman, Phys. Rev. E 61, 4133 (2000)]. We assess the ability of the phenomenological and MCT fits, each with three free fitting parameters, to simultaneously describe the spectra and give reasonable values for the $\alpha$-relaxation time $\tau_{\alpha}$.
\end{abstract}

PACS numbers: 64.70.Pf, 78.35.+c, 67.40.Fd

\section{INTRODUCTION}

Brillouin scattering spectroscopy has been one of the experimental techniques widely applied to the study of glass-forming materials in the continuing effort to achieve a complete understanding of the liquid-glass transition. The evolution of the Brillouin spectrum with temperature reflects the interaction of longitudinal sound waves $\left(q \sim 10^{5} \mathrm{~cm}^{-1}\right)$ with structural relaxation. Typically, as $T$ decreases from above the melting temperature $T_{M}$ to below the glass-transition temperature $T_{G}$, the Brillouin linewidth $\Delta \omega_{B}$ first increases, passes through a maximum, and then decreases again, while the Brillouin peak position $\omega_{B}$ shifts monotonically to higher frequencies, with the increase occurring predominantly in the temperature range where $\Delta \omega_{B}$ is biggest.

In the earliest experiments, only the temperature dependence of the Brillouin shift and linewidth were studied. Subsequently, the full spectrum was analyzed and compared with theoretical predictions derived primarily from generalized hydrodynamics models. Either the Mountain formalism [Mountain 1968] or a generalized damped harmonic oscillator model were used in which the memory function, i.e. the frequency-dependent part of the longitudinal viscosity, represents the structural relaxation dynamics. Generally, acceptable fits could be obtained using an exponential or stretched-exponential empirical memory function $m(t)$ (or a Cole-Davidson memory function $m[\omega]$ ), with the relaxation time $\tau(T)$ treated as an adjustable fitting parameter. However, the $\tau(T)$ values determined from such fits were usually found to increase much more slowly with decreasing $T$ at low temperatures than the values determined with other techniques such as dielectric spectroscopy or ultrasonics.

The origin of this apparent disagreement can be attributed to the use of these " $\alpha$-relaxation only" models for $m(t)$ which ignore the fast part of the relaxation process preceding the final $\alpha$-relaxation. The need for an "additional fast contribution" to $m(\omega)$ was first noted by Loheider et al [Loheider 1990] (also see [Cummins 1994]). Several empirical approaches to extending $m(\omega)$ or $m(t)$ to include the missing fast $\beta$ relaxation have been described in the literature, including: (1) adding a second Debye, Cole-Cole, or Cole-Davidson function to the Cole-Davidson $\alpha$-relaxation term [Soltwisch 1998] [Monaco 2000, Monaco 2001]; (2) extracting a memory function including both $\alpha$ and $\beta$ relaxation from depolarized backscattering spectra and using it for fitting the Brillouin spectra [Cummins 1994] (a procedure that succeeded for CKN but not for other materials); (3) taking $m(t)$ as the sum of a stretched exponential plus a damped high-frequency oscillatory term [Lebon 1997]; and (4) adding a term $B \omega^{a}$ to the Cole-Davidson function to approximate the critical-decay component of the fast $\beta$-relaxation predicted by the mode coupling theory (MCT) [Du 1994, 1996, Cummins 1994, Pick 2000]. This additive "hybrid model" approach, initially suggested by Götze [Gotze 1993], has recently been shown to provide excellent fits to depolarized backscattering spectra of toluene [Wiedersich 2000] and has also been used in the analysis of depolarized Brillouin spectra of metatoluidine [Pick 2000].

With the additional fast relaxation included through any of these approaches, the $\alpha$-relaxation time $\tau_{\alpha}$ is found to increase much more rapidly with decreasing temperature than it does with $\alpha$-only models, consistent with other experimental measurements. However, while this approach can resolve the $\tau(T)$ disagreement, it usually requires additional fitting parameters and does not explain the origin of the structural relaxation dynamics that it describes. Furthermore, various authors have chosen quite different empirical fitting functions and have 
usually obtained good fits to Brillouin spectra, suggesting that this procedure is not able to distinguish effectively between different models.

Another approach to the analysis of Brillouin scattering spectra utilizes the mode coupling theory (MCT) in which the slowing down of structural relaxation with decreasing $T$ arises naturally from nonlinear interactions among density fluctuations. A major advantage of this approach is that both the $\alpha$ and $\beta$ regions of the relaxation dynamics occur spontaneously in the solutions of the MCT equations. However, while MCT can provide a quantitative microscopic description of liquid dynamics in principle, it requires the intermolecular potential [or the static structure factors $S(q)$ ] as input, and this information is not generally available for most real glassforming materials. Therefore, MCT analyses are usually restricted to comparison with asymptotic predictions in which the detailed microscopic interaction information is absorbed into a single parameter, the exponent parameter $\lambda$. Alternatively, a simplified "schematic MCT" can be used in which the full set of density correlators is replaced by a small number (usually one or two), and the coupling constants are treated as adjustable fitting parameters. This schematic approach captures the basic mechanism underlying the MCT more fully than the asymptotic analyses, but sacrifices the material-specific microscopic interpretation of the parameters.

A convenient schematic MCT model, initially introduced by Sjögren [Sjögren 1986], uses two correlators: $\phi(t)$ for the "system" and $\phi_{s}(t)$ for the "probe", i.e. the variable being measured in a particular experiment. In this Sjögren model, the interesting critical behavior occurs in $\phi(t)$ while the temperature dependence of $\phi_{s}(t)$ results from its being coupled to $\phi(t)$. This model was successfully used to analyze depolarized lightscattering spectra of glycerol [Franosch 1997] and OTP [Singh 1998]. Rufflé et al [Rufflé 1999] showed that Brillouin scattering spectra of $\mathrm{NaLi}\left(\mathrm{PO}_{3}\right)_{2}$ could also be analyzed with the Sjögren model, using parameters for the system correlator $\phi(t)$ determined from inelastic neutron scattering spectra.

Recently, Götze and Voigtmann have extended the Sjögren model by including hopping parameters $\Delta$ and $\Delta_{s}$ in the equations of motion for both $\phi(t)$ and $\phi_{s}(t)$, which allows the analysis to be extended to low temperatures where relaxation is dominated by activated hopping processes. They analyzed dielectric, depolarized light scattering, and neutron scattering data for propylene carbonate (PC), using a single set of system parameters for $\phi(t)$, optimized simultaneously for all the experiments [Gotze 2000]. This result suggests the possibility of analyzing PC Brillouin scattering spectra using the system parameters already determined by Götze and Voigtmann. Moreover, if the probe hopping parameter $\Delta_{s}$, frequency $\Omega_{s}$, and damping $\nu_{s}$ are taken as fixed by the depolarized light scattering fits, then the analysis will include only one adjustable MCT fitting parameter, the coupling constant $V_{s}$, which determines the strength of coupling between $\phi(t)$ and $\phi_{s}(t)$. The fits should then simultaneously describe the Brillouin spectra and also determine the value of the structural relaxation time $\tau_{\alpha}$. Such an analysis could provide a stringent test of the utility of MCT for the analysis of Brillouin scattering spectra. A test of this procedure was the principal motivation for the present Brillouin scattering study of PC.

We describe the experiments in Sec. II. We analyze the data in Secs. III and IV using two empirical memoryfunction models, first a conventional $\alpha$-relaxation-only Cole-Davidson (C.D.) function and then the hybrid model. (A preliminary version of the C.D. and hybrid fits was presented by Frank [Frank 2000].) In Sec. IV we also include a discussion of the role of thermal diffusion, followed by a second hybrid model analysis in which the C.D. stretching parameter $\beta$ is constrained to be equal to the MCT critical exponent $b$. In Sec. V we present our analysis of the spectra with the two-correlator extended schematic MCT approach. In Sec. VI, we provide discussion and conclusions including a brief evaluation of the ability of these and related approaches to extract meaningful information about structural relaxation dynamics from the analysis of Brillouin scattering spectra.

\section{EXPERIMENT}

\section{A. Material}

Propylene carbonate $\left(\mathrm{PC} ; T_{G}=160 \mathrm{~K}\right)$ is a molecular glassforming material that has been extensively studied with a wide range of experimental techniques including Brillouin scattering [Elmroth 1992; Du 1994]. In Table I we list the principal properties of $\mathrm{PC}$ that are relevant for the present study.

A recent dielectric spectroscopy study of PC by Schneider et al [Schneider 1999] determined the complex dielectric constant $\epsilon(\omega)$ over a frequency range of 18 decades for temperatures from $153 \mathrm{~K}$ to $293 \mathrm{~K}$. Cole-Davidson fits to the $\alpha$ peaks in the $\epsilon^{\prime \prime}(\omega)$ spectra produced the $\tau_{\alpha}^{\epsilon}(T)$ values shown in Fig. 1 by the open circles. Schneider et al carried out fits to these values with several empirical fitting functions. Their extended free volume fit is shown in Fig. 1 by the solid line.

Du et al [Du 1994] analyzed the $\alpha$ peaks in $\chi^{\prime \prime}(\omega)$ spectra, obtained from their depolarized PC backscattering spectra, with a Kohlrausch function. The resulting $\tau_{\alpha}^{D L S}$ values, shown in Fig. 1 by squares, have temperature dependence similar to the dielectric $\tau_{\alpha}^{\epsilon}(T)$ although they are approximately three times smaller. 


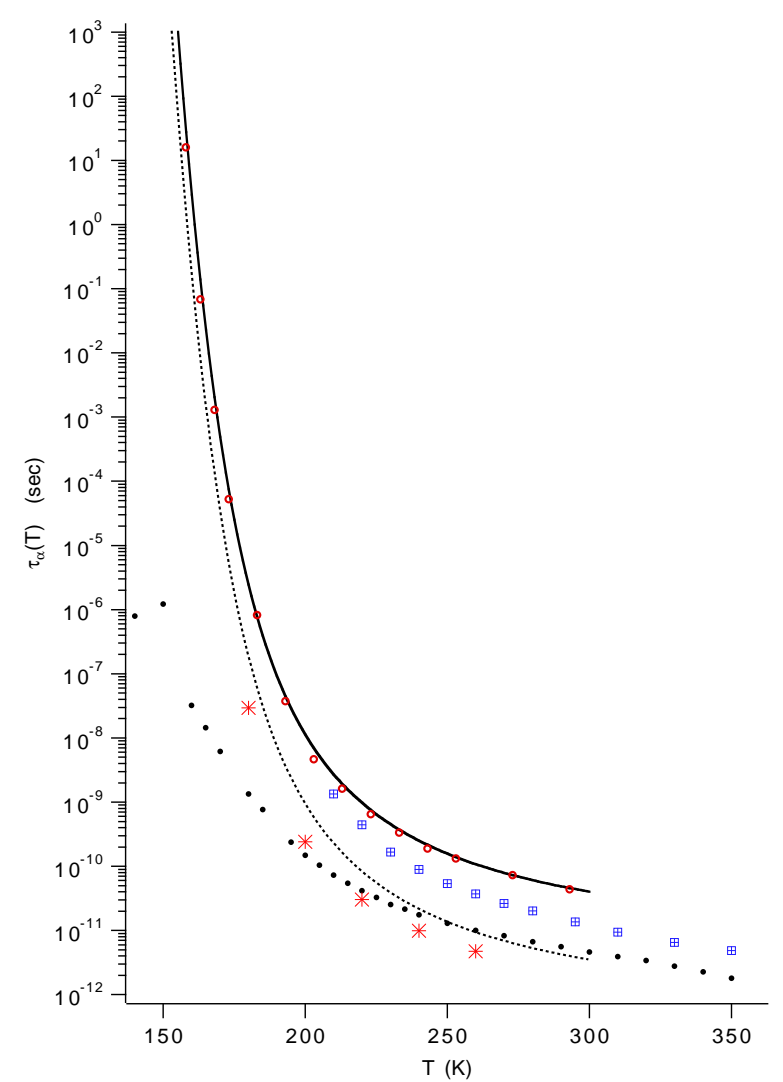

FIG. 1. (TAU). Propylene carbonate (PC) relaxation time $\tau_{\alpha}(T)$. Open circles and solid line: dielectric measurements and extended free-volume fits [Schneider 1999]. Squares: depolarized light scattering spectra [Du 1994]. Solid circles: Brillouin scattering $\tau_{\alpha}^{C D}(T)$ from Cole-Davidson fits (Sec. III). Broken line: dielectric free volume fit multiplied by 0.085 to match Brillouin $\tau_{\alpha}(T)$ in the region of the maximum Brillouin linewidth. $*: \tau_{\alpha}^{M C T}$ obtained from the extended schematic MCT fits.

The analysis of our Brillouin scattering spectra using a Cole-Davidson memory function, described in the next section, resulted in the $\tau_{\alpha}^{C D}$ values shown by the solid circles in Fig. 1. As mentioned in the Introduction, at low temperature these values do not increase as rapidly with decreasing temperature as the dielectric and backscattering results, suggesting that the Cole-Davidson function provides an incomplete representation of structural relaxation. (At $T=T_{G}, \tau_{\alpha}^{C D}$ has only reached $\sim 30 \mathrm{ns,}$ while typically $\tau_{\alpha}\left(T_{G}\right)$ is $\sim 100 \mathrm{~s}$.)

In our $90^{\circ} \mathrm{PC}$ Brillouin scattering experiment, the maximum Brillouin linewidth occurs at $T \sim 230 \mathrm{~K}$. At that temperature the peak of the $\alpha$ relaxation coincides with the Brillouin line, so $\omega_{B} \tau_{\alpha}^{L A} \approx 1$ which gives $\tau_{\alpha}^{L A}$ $(230) \sim 3.2 \times 10^{-11} \mathrm{sec}$, a value $\sim 12$ times smaller than the $\tau_{\alpha}^{\epsilon}$ of Schneider et al. The difference presumably arises from the fact that $\tau_{\alpha}^{L A}$ characterizes longitu- dinal displacement dynamics while $\tau_{\alpha}^{\varepsilon}$ characterizes orientational dynamics. In Fig. 1, the lower line is the free volume fit of Schneider et al divided by a factor of 11.75 to match $\tau_{\alpha}^{L A}$ in this region:

$$
\log _{10}\left(\tau_{\alpha}^{L A}\right)=-A+B /\left\{T-T_{0}+\left[\left(T-T_{0}\right)^{2}+C T\right]^{1 / 2}\right\}
$$

with $A=12.56, B=309, C=4.82$, and $T_{0}=162 \mathrm{~K}$ ( $\tau_{\alpha}^{L A}$ in seconds). In constructing memory functions for data analysis beyond the Cole-Davidson model, we will use Eq. (1) to fix the $\alpha$-relaxation time of the longitudinal acoustic mode $\tau_{\alpha}^{L A}$.

\section{B. Sample Preparation}

Propylene carbonate (anhydrous, $99.7 \%$ ) was purchased from Sigma-Aldrich. PC was loaded in a glove box under dry nitrogen atmosphere into a distillation flask fitted with a stopcock. The flask was then transferred to a vacuum distillation system and distilled at $\sim 110 \mathrm{C}$ into glass sample cells that were flame sealed under vacuum. A sample cell was installed in an Oxford LN2 coldfinger cryostat with an ITC-4 temperature controller for the Brillouin scattering experiments.

\section{Brillouin scattering experiments}

Spectra were collected in both $90^{\circ} \mathrm{VV}$ geometry and $174^{\circ} \mathrm{VH}$ near-backscattering geometry with a Sandercock 6-pass tandem Fabry-Perot interferometer at temperatures from $140 \mathrm{~K}$ to $350 \mathrm{~K}$ in $5 \mathrm{~K}$ or $10 \mathrm{~K}$ steps. The $514.5 \mathrm{~nm}$ single-mode argon laser power at the sample was typically $170 \mathrm{~mW}$. The interferometer finesse was $\sim 90$; its contrast was better than $10^{7}$. Complete sets of spectra were collected with mirror separations $d=2,5$, and $10 \mathrm{~mm}$ for $\mathrm{VV}$ and $d=2 \mathrm{~mm}$ and $10 \mathrm{~mm}$ for VH. Spectra were accumulated during 1 to 2 hour runs. For the $d=10 \mathrm{~mm}$ separation spectra two 2-hour runs were collected and averaged to improve the signal-to-noise ratio. In Fig. 2 we plot the Stokes side of the three VV spectra (a) and the two VH spectra (b) for $T=220 \mathrm{~K}$. (The dark counts have already been subtracted.)

In order to test for possible experimental artifacts, $I_{I S O}(\omega) \mathrm{PC}$ Brillouin spectra at several temperatures were collected at both the Technical University of $\mathrm{Mu}-$ nich and at the City College of New York with different samples, different tandem Fabry-Perot spectrometers, and different experimental procedures. The spectra obtained were in very good agreement. The spectra shown in this work were all collected in New York. 

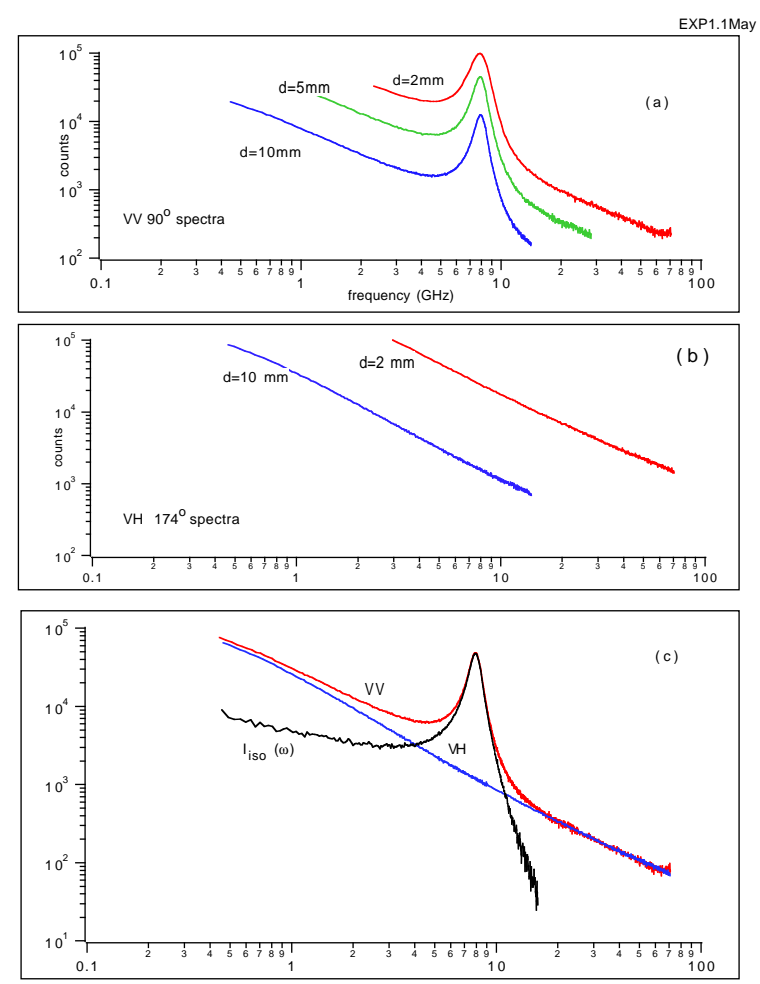

FIG. 2. (EXP1). (a) Stokes sides of $220 \mathrm{~K} \mathrm{PC} 90^{\circ} \mathrm{VV}$ Brillouin spectra with $d=2,5$, and $10 \mathrm{~mm}$. (b) $174^{\circ}$ VH spectra with $d=2 \mathrm{~mm}$ and $10 \mathrm{~mm}$. (c) The composite $90^{\circ} \mathrm{VV}$ spectrum obtained by shifting the $2 \mathrm{~mm}$ and $5 \mathrm{~mm}$ spectra of (a) to optimize overlap with the $10 \mathrm{~mm}$ spectrum, the composite VH spectrum obtained similarly and then shifted to optimize overlap with the VV spectrum for frequencies above $30 \mathrm{GHz}$, and the difference spectrum $I_{I S O}(\omega)$ obtained by subtraction. The background from dark counts has already been subtracted in the spectra of (a) and (b).

\section{Data reduction: extraction of the density fluctuation spectra $I_{I S O}(\omega)$}

The VV polarized Brillouin spectra contain contributions from density fluctuations $\left[I_{I S O}(\omega)\right]$ and also from orientational dynamics and collision-induced scattering $\left[I_{A N I}(\omega)\right]$. Depolarized $(\mathrm{VH})$ backscattering spectra are due to $I_{A N I}(\omega)$ only, and can therefore be used to remove the $I_{A N I}(\omega)$ contribution from the $\mathrm{VV}$ spectra.

Fioretto et al [Fioretto 1999] [Monaco 2001] have carried out the subtraction by combining spectra obtained with different mirror separations to obtain broadband VV and VH spectra, and scaling the VH spectra to overlap the VV spectra for frequencies above $\sim 40 \mathrm{GHz}$ where $I_{I S O}(\omega)$ should be negligible. Subtracting the scaled
$I_{V H}(\omega)$ from the $I_{V V}(\omega)$ then provides $I_{I S O}(\omega)$. We have also followed this procedure.

The Stokes sides of the $d=2$ and $10 \mathrm{~mm} 220 \mathrm{~K} \mathrm{VV}$ spectra, shown in Fig. 2(a), were rescaled vertically to optimize overlap with the $d=5 \mathrm{~mm} \mathrm{GHz}$ spectrum, producing the composite $90^{\circ} I_{V V}(\omega)$ spectrum shown in Fig. 2(c). The $\theta=184^{\circ} I_{V H}(\omega)$ spectra were similarly combined, and the composite $I_{V H}$ spectrum was again scaled vertically to match the $I_{V V}$ spectrum in the 30 $50 \mathrm{GHz}$ region. Finally, the scaled $I_{V H}$ spectrum was subtracted from the $I_{V V}$ spectrum to produce the difference spectrum $I_{I S O}(\omega)$ as shown in Fig. 2(c).

The full set of $I_{I S O}(\omega)$ spectra for temperatures from $140 \mathrm{~K}$ to $350 \mathrm{~K}$ obtained with this procedure is shown in Fig. 3. For each set of VV spectra, we also recorded an instrument profile spectrum with $d=10 \mathrm{~mm}$. The width of the instrumental profile was typically FWHH $=0.17 \mathrm{GHz}$. To reduce additional Brillouin peak broadening due to the finite input aperture (which leads to collecting light scattered at slightly different angles), the input aperture was reduced sufficiently to decrease the additional broadening at the Brillouin frequency to less than the instrumental width. This additional geometrical broadening (typically $\lesssim 0.13 \mathrm{GHz}$ ) was then calculated for every temperature from the known collection geometry and convoluted into the instrument function. In the fitting procedures described in this paper, the instrument function so obtained was convoluted with the theoretical spectrum for comparison with the $I_{I S O}(\omega)$ spectrum. The fits were carried out with a conventional nonlinear least-squares fitting program (NLLSQ).

We first fit each Brillouin peak in the $d=10 \mathrm{~mm}$ $V V$ spectra to the damped harmonic oscillator func-

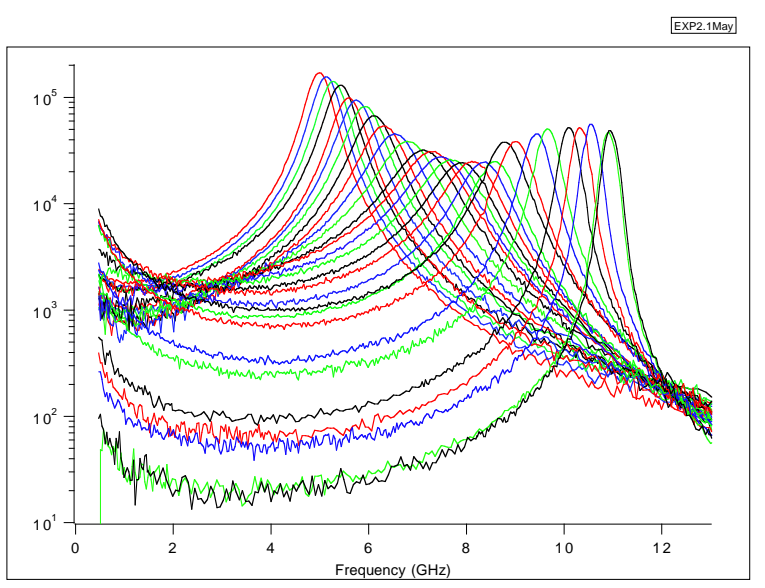

tion

FIG. 3. (EXP2). Complete set of composite $I_{I S O}(\omega)$ spectra for 28 temperatures from $140 \mathrm{~K}$ to $350 \mathrm{~K}$ obtained by the procedure shown in Fig. 2. $T=140,150$, $160,165,170,180,185,195,200,205,210,215,220$, $225,230,235,240,250,260,270,280,290,300,310$, $320,330,340$, and $350 \mathrm{~K}$, from right to left. 


$$
\begin{array}{r}
I(\omega)=\frac{I_{0}}{\omega} \mathcal{I} m\left[\omega^{2}-\omega_{B}^{2}-i \omega \gamma_{B}\right]^{-1} \\
=\frac{I_{0} \gamma_{B}}{\left[\omega^{2}-\omega_{B}^{2}\right]^{2}+\left[\omega \gamma_{B}\right]^{2}}
\end{array}
$$

including convolution with the instrument function. In Fig. 4 the resulting values of $\omega_{B}(a)$ and $\gamma_{B}(b)$ are shown by the points. For a simple liquid, both $\omega_{B}$ and $\gamma_{B}$ would have simple monotonic temperature dependence. The observed temperature dependence of $\omega_{B}$ and $\gamma_{B}$, as noted in the introduction, is the signature of structural relaxation dynamics as seen in Brillouin scattering. The maximum in $\gamma_{B}$ at $T \sim 230 \mathrm{~K}$ indicates that the $\alpha$ peak moves through the Brillouin line at that temperature.

\section{DATA ANALYSIS 1: THE COLE-DAVIDSON MODEL}

\section{A. The memory function approach}

The isotropic Brillouin spectrum $I_{I S O}(\omega)$ is usually attributed to density fluctuations $\rho_{q}(t)$ where the scattering vector $q=(2 \pi n / \lambda) \sin (\theta / 2)$. The spectrum consists of a triplet: the central thermal diffusion mode (which is generally located at frequencies too low to observe in these experiments and will be ignored in most of the fits) and the Brillouin doublet. The spectrum is conventionally described by

$$
I(\omega)=\frac{I_{0}\left[\gamma_{0}+m^{\prime \prime}(\omega)\right]}{\left[\omega^{2}-\omega_{0}^{2}+\omega m^{\prime}(\omega)\right]^{2}+\left[\omega \gamma_{0}+\omega m^{\prime \prime}(\omega)\right]^{2}}
$$

[The particular Laplace transform convention used to derive Eq. (3) is discussed in the Appendix.]

Equation (3) is very general; $m(\omega)=m^{\prime}(\omega)+i m^{\prime \prime}(\omega)$ is the Laplace transform of the memory function $m(t)$ which can be constructed to include structural relaxation, thermal diffusion, and translation-orientation coupling for liquids of anisotropic molecules [Chung 1971; Boon 1980; Scopigno 2000]. Usually, however, $m(t)$ represents the relaxing longitudinal viscosity, first introduced by Mountain [1968] to describe the damping of sound waves by interaction with internal degrees of freedom of the molecules, and later utilized to represent the interaction of sound waves with structural relaxation. (Both mechanisms may contribute, however, as discussed below.) In the long-wavelength limit, $\omega_{0}=C_{0} q$ where $C_{0}$ is the limiting low-frequency adiabatic sound velocity, and $\gamma_{0}$ is the "regular" sound attenuation coefficient. Note that Eq. (3) is the equation for a damped harmonic oscillator with a frequency-dependent damping function $\gamma(\omega)=\gamma_{0}+m(\omega)$.

Ideally, the $I_{I S O}(\omega)$ spectra should be analyzed to yield $m(\omega)$ directly. But in practice this is not possible because the spectra only cover a range of two decades at most, while $m(\omega)$ extends over many decades. Also, the spectra are dominated by the Brillouin components [which would be the complete spectrum of Eq. (3) if $m(\omega)=0]$, and the modification of $I(\omega)$ produced by inclusion of $m(\omega)$ is not very sensitive to the detailed form of $m(\omega)$. Therefore, data analysis almost always proceeds by selecting a parameterized model for $m(\omega)$ and varying the parameters at each temperature to optimize the fits.

The primary requirements on $m(\omega)$ are that $m^{\prime}\left(\omega_{B}\right)$, the real part of $m(\omega)$ at the frequency of the Brillouin peak, must be adjusted to shift the Brillouin peak from $\omega_{0}$ to $\omega_{B}$; and $m^{\prime \prime}\left(\omega_{B}\right)$, the imaginary part of $m(\omega)$ at the frequency of the Brillouin peak, must be adjusted so that $\gamma_{0}+m^{\prime \prime}\left(\omega_{B}\right)$ will give the correct Brillouin linewidth. If these two conditions are simultaneously satisfied, then the particular form chosen for $m(\omega)$ will only influence the details of the lineshape. Therefore the essential requirement is that $m^{\prime \prime}\left(\omega_{B}\right) / m^{\prime}\left(\omega_{B}\right)$ must have the "correct" value. Nevertheless, this is not a trivial requirement since $m^{\prime}(\omega)$ and $m^{\prime \prime}(\omega)$ are connected by Kramers-Kronig relations.

The prototype memory function is the exponential $e^{-t / \tau}$, multiplied by a coupling constant $\Delta^{2}$. (It is usually called Debye relaxation although it was first used by Maxwell in his theory of viscoelasticity.) The simplest memory function $m(t)$ for structural relaxation that fits various experimental data in the frequency region of $\alpha$ relaxation is the stretched-exponential (KWW) function $e^{-(t / \tau)^{\beta} K}$. An approximation to the Fourier transform of this $m(t)$ is provided by the Cole-Davidson (C.D.) function

$$
m(\omega)=\left(\Delta^{2} / \omega\right)\left[(1-i \omega \tau)^{-\beta}-1\right]
$$

which has frequently been used, together with Eq. (3), to analyze Brillouin-scattering spectra. In practice, the fitting procedure adjusts the coupling constant $\Delta^{2}$ so that the real part of $m(\omega)$ produces the correct $\omega_{B}$ for the Brillouin peak, while $\tau$ is adjusted to give the correct Brillouin linewidth.

There are several problems that should be noted in carrying out this analysis, as well as analyses with more elaborate memory functions.

(1) Many glassforming materials are molecular liquids, and internal vibrational modes may also contribute to $m(\omega)$, as originally proposed by Mountain [1968]. Recently, Monaco et al ([Monaco 2000]) have carried out a Brillouin scattering study of OTP from which they conclude, for OTP, that the fast part of the relaxation process is entirely due to intramolecular vibrational modes. The assignment results from the presence of additional low frequency structure (Mountain mode) in VV, but not VH, spectra, in both the glass and the crystal. This conclusion has recently found additional support from a molecular dynamics study of OTP [Mossa 2001]. There is no evidence, however, that it applies to other materials such as PC, and it will not be considered here. 
(2) The identification of $I_{I S O}(\omega)$ with density fluctuation rests on the assumption that scattering due to orientational fluctuations, which is typical for molecular glassforming materials, is eliminated by the subtraction of $I_{a n i}(\omega)$ from $I_{V V}(\omega)$ described in the previous section. However, two consequences of anisotropy may remain after subtraction. First, since longitudinal current involves both compression and shear, rotation-translation coupling modifies the longitudinal current correlator [which determines the spectrum of $\rho_{q}(t)$ ] as shown in Eq. (A.14) of Ref. [Dreyfus 1999]. Second, as noted recently by Latz and Letz [Latz 2000], because of this coupling the orientational dynamics also reflect the longitudinal acoustic mode, and this part of the orientational dynamics is not removed by subtraction. Therefore, both density fluctuations and orientational dynamics may contribute to the $I_{I S O}(\omega)$ spectra.

While the extent of these corrections due to molecular anisotropy is not yet clear, preliminary simulations, carried out in collaboration with R.M. Pick, indicate that they are relatively minor. We will therefore use the conventional memory function formalism in this paper, recognizing that the values of the parameters used to describe the frequency-dependent longitudinal viscosity may reflect some aspects of translation-rotation coupling and molecular anisotropy and may also include some contribution from intramolecular vibrational dynamics.

\section{B. Cole-Davidson Fits}

We first analyzed the $I_{I S O}(\omega)$ spectra of Fig. 3 using Eq. (3) with the C.D. memory function $m(\omega)$ of Eq. (4). The fit results, for a subset of the spectra, are shown in Fig. 5 [note (IIIB.1)]. As often found in the past, excellent fits can be obtained if all the parameters are kept free. However, it is preferable to fix as many parameters as possible using independently determined values, since the parameters are rather strongly coupled. (This coupling can also lead to unstable parameter values in the fit results.) Here we consider each of the relevant parameters separately.

(1) $\beta$. The fits are relatively insensitive to this parameter. We took $\beta=0.68$ (corresponding to $\beta_{K}=0.77$ ) as found in the analysis of depolarized backscattering spectra by Du et al [Du 1994].

(2) $\omega_{0}(T)$. Since $\omega_{0}=C_{0} q$ where $C_{0}$ is the limiting low-frequency adiabatic sound velocity and $q$ is the scattering wavevector $(4 \pi n / \lambda) \sin (\theta / 2), \omega_{0}(T)$ can be fixed if $C_{0}(T)$ and the refractive index $n(T)$ are known. $C_{0}(T)$ was previously measured by ultrasonic experiments at $5 \mathrm{MHz}$ and $15 \mathrm{MHz}$ by $\mathrm{Du}$ et al [Du 1994] which gave $C_{0}(T)=2.507 \times 10^{5}-361.2 T \mathrm{~cm} / \mathrm{sec}$.

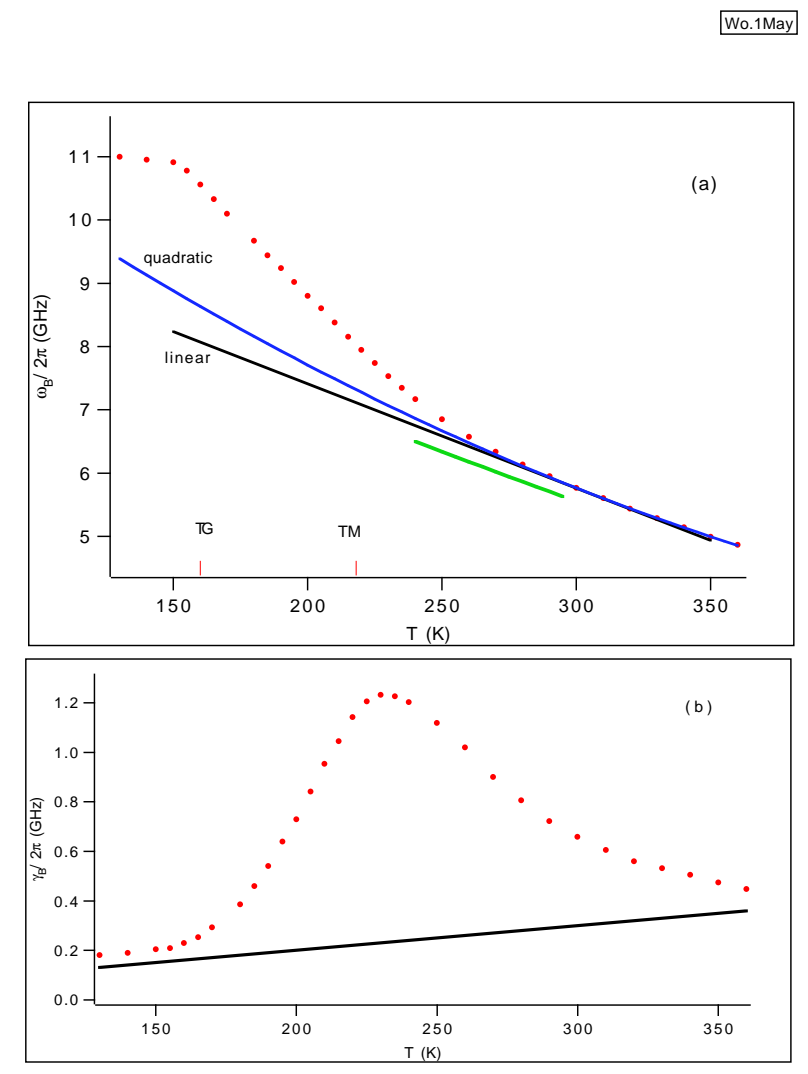

FIG. 4. (Wo). Fits of the $d=10 \mathrm{~mm} 90^{\circ} \mathrm{VV}$ Brillouin peaks to Eq. (2) including convolution with the instrument function. (a) $\omega_{B} / 2 \pi(\mathrm{GHz})$ (circles). Short solid line: $\omega_{0}$ from ultrasonic and refractive index data; long solid line: $\omega_{0}$ increased by $3.5 \%$ to match $\omega_{B}$ in the high-temperature region. Solid curved line: quadratic fit $\omega_{0}(T)=13.211-3.291 \times 10^{-2} \mathrm{~T}+2.6973 \times 10^{-5} \mathrm{~T}^{2}$. (b) $\gamma_{B} / 2 \pi(\mathrm{GHz})$ (circles). Solid line: $\gamma_{0}=T / 1000$.

However, the ultrasonic data only cover the range 240 to $293 \mathrm{~K}$. At lower temperatures, there is significant dispersion in the ultrasonic sound speed, while higher temperature measurements are technically difficult. The refractive index $n(T)=1.5314-3.752 \times 10^{-4} T$ (see Table I) corresponds to the sodium $D$ line and may be different at $514.5 \mathrm{~nm}$. The $\omega_{0}(T)$ values implied by these measurements are shown in the upper panel of Fig. 4, by the short solid line. A linear extrapolation of these values, shifted up by $3.5 \%$, is indicated by the long solid line labeled "linear": $\omega_{0}(T) / 2 \pi=(10.71-0.0165 \times T) \mathrm{GHz}$. The shift, which is within the experimental errors of the $C_{0}(T)$ and $n(T)$ data, was chosen so that at high temperatures the Brillouin peak frequency $\omega_{B}$ is equal to $\omega_{0}$, as expected. Note, however, that the high- $T \omega_{B}$ values suggest some small upward curvature away from the linear extrapolation above $320 \mathrm{~K}$.

Initially, fits were carried out with this linear- $T$ estimate of $\omega_{0}(T)$. However, the fits were not all satisfactory, 
in part because of the high- $T$ curvature in $\omega_{B}$ seen in Fig. 4. For materials where $C_{0}(T)$ measurements extend over larger temperature ranges, there can be significant upward curvature to $C_{0}(T)$. We therefore tried fitting $\omega_{0}(T)$ to the six highest temperature $\omega_{B}(T)$ values plus one $\omega_{0}(T)$ value obtained by a free C.D. fit at $230 \mathrm{~K}$ where the Brillouin linewidth is largest. The result was the quadratic $\omega_{0}(T)$ function shown by the upper solid line in Fig. 4 labeled "quadratic":

$$
\omega_{0}(T)=13.211-3.291 \times 10^{-2} T+2.6973 \times 10^{-5} T^{2}
$$

which was used in all subsequent fits.

(3) $\gamma_{0}(T)$. This term is conventionally included to represent "regular" damping of the sound waves by anharmonic processes not related to structural relaxation. It can also represent a first frequency-independent approximation for the fast part of the structural relaxation not included in $\alpha$-relaxation-only models such as the C.D. function. Usually, $\gamma_{0}$ is taken as a temperatureindependent constant, fixed from the Brillouin linewidth at low temperatures.

However, since anharmonic damping processes often tend to become stronger with increasing temperature, $\gamma_{0}$ may increase with $T$. Also, at temperatures well above the temperature of the maximum Brillouin linewidth, the linewidth due to $\gamma_{0}$ plus structural relaxation is approximately given by $\Delta \omega_{B} \approx \gamma_{0}+\Delta^{2} \tau \beta$. Fits with constant $\gamma_{0}$ often give $\Delta^{2}$ values that increase at high temperatures, a result that appears unphysical. This apparent increase in $\Delta^{2}$ can be avoided by allowing $\gamma_{0}$ to increase with increasing $T$. To avoid introducing additional fitting parameters we arbitrarily assumed $\gamma_{0}$ to be a linear function of $T$, and took $\gamma_{0}(T)=T / 1000$ as shown by the solid line in the lower panel of Fig. 4.

(4) $\Delta^{2}(T)$. Since at high temperatures $\omega_{B}=\omega_{0}$ while $\Delta \omega_{B}=\gamma_{0}+\Delta^{2} \tau \beta$, the effective fitting parameter for $\Delta \omega_{B}$ is the product $\Delta^{2} \tau$. So if $\Delta^{2}$ and $\tau$ are both free parameters, the fits tend to be unstable. We therefore carried out the fits for temperatures up to $235 \mathrm{~K}$ with the three free fitting parameters: $\Delta^{2}, \tau$, and the scale factor $I_{0}$. For $T>235 \mathrm{~K}, \Delta^{2}$ was fixed at its value at $\sim 235 \mathrm{~K}$, and the fits were carried out with only $\tau$ and $I_{0}$ free. The parameters $\tau(T)$ and $\Delta^{2}(T)$ obtained from these fits are shown in Table II along with the reduced $\chi^{2}$ value for each fit. The C.D. fits, shown in Fig. 5, are satisfactory although better fits could be obtained by keeping more parameters free, especially $\omega_{0}$.

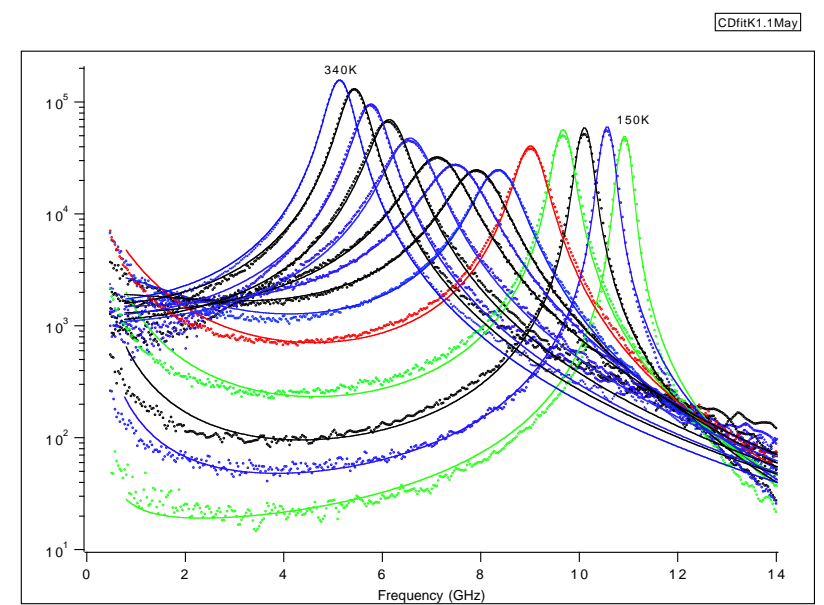

FIG. 5. (CDFit). Cole-Davidson fits to the $I_{I S O}(\omega)$ spectra of Fig. 3 to Eqs. (3) and (4), with $\beta=0.68$, $\omega_{0} / 2 \pi=13.211-3.291 \times 10^{-2} \mathrm{~T}+2.6973 \times 10^{-5} \mathrm{~T}^{2}$, $\gamma_{0} / 2 \pi=T / 1000$. The fits are shown for $T=150,160$, 170, 180, 195, 210, 220, 230, 240, 260, 280, 300, 320, and $340 \mathrm{~K}$.

However, the major point of the C.D. fits, as shown by the solid circles in Fig. 1, is that for temperatures below $\sim 230 \mathrm{~K}, \tau_{\alpha}^{C D}$ determined from these fits does not increase very rapidly with decreasing $T$. At $T=T_{G}=$ $160 \mathrm{~K}, \tau_{\alpha}^{C D}$ found from the C.D. fits is only $\sim 30 \mathrm{~ns}$, compared to $\tau \sim 100 \mathrm{~s}$ found in other experiments. This disagreement is generally recognized as indicating that the C.D. model (or any $\alpha$-relaxation-only model) for $m(\omega)$ is incomplete. Once the $\alpha$ peak moves well below the frequency of the Brillouin peak, $m^{\prime \prime}(\omega)$ of the C.D. function [with reasonable $\tau(T)$ values] decreases rapidly with decreasing temperature, while the shape of the spectrum indicates that a significant contribution from $m^{\prime \prime}(\omega)$ is still present in the region of the Brillouin peak.

The fact that the C.D. function is an incomplete representation of the structural relaxation dynamics can also be seen directly in the PC dielectric data of Schneider et al [Schneider 1999]. Their Fig. 2 shows C.D. fits to the dielectric data which are excellent for the $\alpha$ peak, but which fall below the experimental data in the highfrequency wing.

\section{DATA ANALYSIS 2: INCLUDING THE FAST $\beta$ RELAXATION}

The $\tau(T)$ values obtained from the Cole-Davidson fits exhibit temperature dependence at low temperatures that disagrees strongly with the results of other experiments, as seen in Fig. 1. As mentioned in the introduction, this disagreement is a characteristic failure of $\alpha$-relaxation-only models for $m(\omega)$, which indicates the 
need for extending $m(\omega)$ to include fast relaxation processes. Several authors have proposed models that extend the C.D. model empirically by adding another phenomenological term to Eq. (4), e.g. a Cole-Cole term [Soltwisch 1998], a damped oscillator [Lebon 1997], or a Lorentzian [Monaco 2000B]; however, attributing a fast relaxation time $\tau_{\beta}$ to structural relaxation is incompatible with MCT which describes the fast relaxation by power laws in $\omega$ or $t$ for which there is no $\tau_{\beta}$ [Götze 1992]. A major advantage of this approach is that the additional fast C.D. or Lorentzian, with relaxation time $\tau_{\beta} \sim 30 \mathrm{ps,}$ is able to explain the extra "Mountain mode" seen in OTP at low temperatures [Monaco 1999, Fig. 1(a)]. (Recently, however, this feature has been attributed to Mountain's original mechanism of intramolecular dynamics rather than to structural relaxation [Monaco 2000, Mossa 2001].)

\section{A. The hybrid model}

We have utilized a different additive memory function, the hybrid model (mentioned in the introduction), which has been employed in several previous Brillouin scattering studies. We begin with the C.D. function [Eq. (4)] and add a term to $m(\omega)$ proportional to $\omega^{a-1}$ which represents the $t^{-a}$ "critical decay" part of the fast $\beta$ process in MCT. This superposition approximates the two-step relaxation scenario of MCT, and does not introduce another relaxation time $\tau_{\beta}$.

In some previous studies, we have used a related procedure to analyze depolarized backscattering spectra. For orthoterphenyl, we combined extended MCT fits for the $\beta$-relaxation region with $\mathrm{KWW}$ fits for the $\alpha$ peak [Cummins 98]. A comparison of these two hybrid procedures has not yet been attempted.

Two problems arise when the term $t^{-a}$ is added to $m(t)$. First, the resulting $\omega^{a}$ term in $\omega m(\omega)$ extends to arbitrarily high frequencies. This is presumably unimportant for Brillouin scattering, however, since fits to the Brillouin spectra are limited to $\omega / 2 \pi \lesssim 15 \mathrm{GHz}$. Second, $m^{\prime \prime}(\omega)$, which appears in the numerator of $I(\omega)$ in Eq. (2), diverges at low frequencies as $\omega^{a-1}$ resulting in the introduction of a spurious "central peak". To eliminate this artifact, we have included an exponential cutoff in the critical decay part of $m(t)$ [Gotze 2000B]:

$$
m_{c r i t}(t)=B t^{-a} e^{-(t / \tau)}
$$

where $\tau$ is the same $\tau_{\alpha}^{C D}$ of the C.D. function, Eq. (4). (Note that this cutoff factor has not been included in previous analyses based on the hybrid model.)

With this modification, the hybrid memory function becomes

$$
\begin{gathered}
\omega m(\omega)=\Delta^{2}\left[(1-i \omega \tau)^{-\beta}-1\right] \\
+i \omega B \Gamma(1-a)\left(\tau^{-1}-i \omega\right)^{a-1}
\end{gathered}
$$

[see the Appendix for the derivation of Eq. (7).]

At high frequencies where $\omega \tau>>1$, the critical decay part of Eq. (7) becomes

$$
\omega m_{C R}(\omega)=\left[B_{1}+i B_{2}\right] \omega^{a}
$$

where $B_{1}=B \sin [\pi(a-1) / 2] \Gamma(1-a)$ and $B_{2}=$ $B \cos [\pi(a-1) / 2] \Gamma(1-a)\}$. Equation (8) is the familiar high-frequency power-law behavior predicted by MCT.

In Fig. 6 we plot $\omega m^{\prime \prime}(\omega)$ of Eq. (7) with $\tau=0.3 \mathrm{ns,}$ $\beta=0.68$, and $a=0.29$. The broken line is the Cole-Davidson term only, and the lower solid curve is the critical decay term only, including the exponential cutoff. The upper solid curve is the full hybrid memory function $\omega m^{\prime \prime}(\omega)$ of Eq. (7) with $B / \Delta^{2}=0.005$. This figure illustrates how the resulting $\omega m^{\prime \prime}(\omega)$ goes over smoothly from the C.D. $\alpha$-peak behavior at low frequencies to the $\omega^{a}$ behavior at high frequencies, with a minimum between the two regions. The inset to Fig. 6 shows the full $m^{\prime \prime}(\omega)$ with and without the $e^{-t / \tau}$ cutoff, illustrating how the spurious central peak is eliminated by the exponential cutoff.

\section{B. The critical exponent $a$}

In MCT, the function $\omega m^{\prime \prime}(\omega)$ is predicted to have minimum between the high-frequency von-Schweidler wing of the $\alpha$ peak and the critical decay region similar to that in Fig. 6. In the region of this minimum, the MCT interpolation equation gives $\omega m^{\prime \prime}(\omega)$ in terms of the critical exponents $a$ and $b$ by

$$
\begin{aligned}
\omega m^{\prime \prime}(\omega)= & \omega m_{\min }^{\prime \prime}(\omega)\left[b\left(\omega / \omega_{\min }\right)^{a}\right. \\
& \left.+a\left(\omega_{\min } / \omega\right)^{b}\right] /(a+b)
\end{aligned}
$$

where $a$ is the critical exponent and $b$ is the vonSchweidler exponent, representing the high-frequency wing of the $\alpha$ peak.

Since in our hybrid memory function, Eq. (7), the C.D. function for $\omega \tau>>1$ is $\propto \omega^{-\beta}$, the interpolation Eq. (9) with $b=\beta$ will determine an "effective" $a_{\text {eff }}$ to use in Eq. (7) at temperatures high enough for the minimum to be observable (from previous MCT fits, $b \approx 0.50$ while $\beta \sim 0.68)$. At lower $T$, where the minimum has disappeared from the frequency region of the Brillouin spectrum, $\omega m^{\prime \prime}(\omega)$ may have the form of a power law in $\omega$, but since this region no longer corresponds to the asymptotic region of the minimum, the apparent power law behavior of $\omega m^{\prime \prime}(\omega) \propto \omega^{a_{e f f}}$ can produce an $a_{e f f}$ quite different from the critical exponent $a$ itself. 


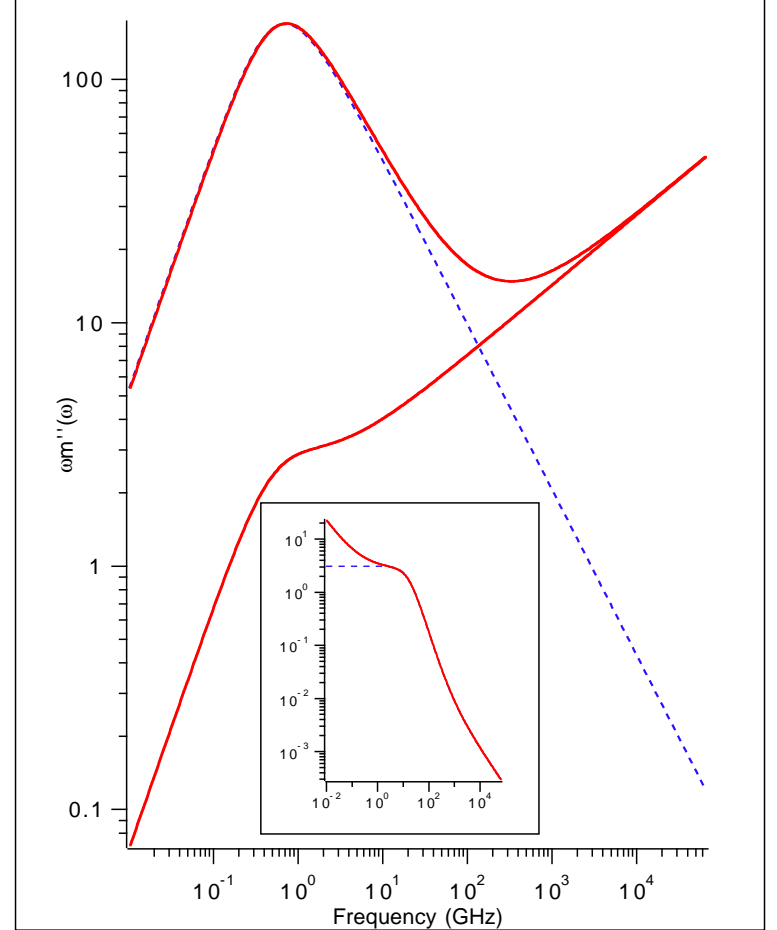

FIG. 6. (HYB1). The hybrid memory function $\omega m^{\prime \prime}(\omega)$ of Eq. (7) with $\tau=0.3 \mathrm{~ns}, \beta=0.68$, and $a=0.29$. The broken line is the C.D. function and the lower solid line is the critical decay term. The ratio of the critical decay to C.D. contributions is $B / \Delta^{2}=0.005$. Inset: $m^{\prime \prime}(\omega)$ vs $\omega$ for the hybrid model [Eq. (7)] without a cutoff (solid line), with $\tau=0.01 \mathrm{~ns}, \Delta=20 \mathrm{GHz}$, and $B=5$; $m^{\prime \prime}(\omega)$ with the cutoff $e^{-t / \tau}$ in Eq. (6) (broken line).

We utilized the depolarized backscattering spectra $I_{V H}(\omega)$, multiplied by $\omega$ to estimate $\omega m^{\prime \prime}(\omega)$ in order to find $a_{e f f}(T)$. In Fig. 7 we show six $\omega I_{V H}(\omega)$ spectra for $T=140,150,160,170,180$, and $185 \mathrm{~K}$. The spectra for $T=170,180$, and $185 \mathrm{~K}$, where the minimum is visible, have been fit to the interpolation equation [Eq. (9)] using $\beta=0.68$ for $b$, while the spectra for $T=140,150$, and $160 \mathrm{~K}$ have been fit to $\omega^{a_{e f f}}$. The values of $a_{e f f}(T)$ found from these fits are:

$\begin{array}{cc}T & a_{\text {eff }} \\ 140 & 0.62 \\ 150 & 0.59 \\ 160 & 0.52 \\ 170 & 0.42 \\ 180 & 0.30\end{array}$

In the fits to our Brillouin spectra, we used these values of $a_{e f f}(T)$ for temperatures up to $180 \mathrm{~K}$ and $a=0.29$ for all higher temperatures.

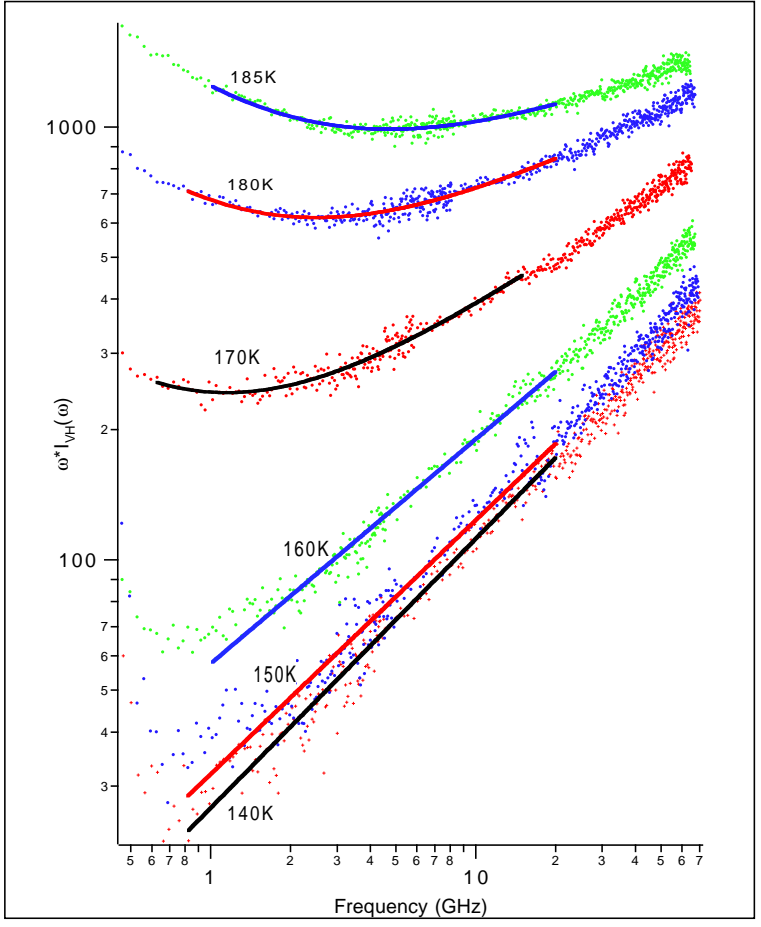

FIG. 7. (HYBFIT2). VH backscattering spectra multiplied by frequency with fits to the MCT interpolation equation [Eq. (9)] (using $b=\beta=0.68)(T=170,180$, $185 \mathrm{~K})$ or the power law $\omega^{a_{e f f}}(T=140,150,160 \mathrm{~K})$. The values of $a_{\text {eff }}$ determined in these fits were used in the low-temperature $I_{I S O}(\omega)$ hybrid model fits shown in Figs 8 and 9.

\section{Minimum free parameter analysis}

We initially tried to fit the spectra with Eq. (3) and the hybrid memory function Eq. (7) with the smallest possible number of free parameters. We fixed $\omega_{0}(T)$ with Eq. (5), fixed $\gamma_{0}(T)=T / 1000$ as in the Cole-Davidson fits, fixed $\tau$ using Eq. (1), fixed $\beta=0.68$, and fixed the ratio $B / \Delta^{2}$, the relative strengths of the critical decay term $(B)$ and Cole-Davidson term $\left(\Delta^{2}\right)$, by the following strategy. For fixed $\tau$, the location of the minimum in the function $\omega m^{\prime \prime}(\omega)$ occurs at a frequency $\omega_{\min }$ that decreases as $B / \Delta^{2}$ increases. We then invoked the MCT result that $\omega_{\text {min }}$ (unlike $\tau_{\alpha}$ ) should be equivalent for all variables, and we adjusted $B / \Delta^{2}$ to make $\omega_{\min }$ fall close to the $\omega_{\min }$ found in the depolarized backscattering PC $\chi^{\prime \prime}(\omega)$ spectra of Du et al [Du 1994]. For $T=190,210$, 230,250 and $270 \mathrm{~K}$, the $\omega_{\min }$ values of $\mathrm{Du}$ et al were found to agree quite well with the minima of the $\omega m^{\prime \prime}(\omega)$ curves computed with Eq. (7) with $B / \Delta^{2}=0.175$. With 
this fixed value of $B / \Delta^{2}=0.175$, there are only two free parameters left in Eqs. (7) and (3): the C.D. coupling constant $\Delta^{2}$, and the overall normalization constant $I_{0}$.

With this severely constrained fit procedure, however, the resulting fits were not generally acceptable. One surprising aspect of the results was that for $T \leq 170 \mathrm{~K}$ the linewidths of the fits became too broad; this problem can be resolved by decreasing $B / \Delta^{2}$.

This indication that the strength of the critical contribution to the memory function (relative to the $\alpha$ peak) may weaken in the temperature range between $T_{C}$ and $T_{G}$ is also supported by the depolarized backscattering spectra, shown in Fig. 7. At temperatures below $T=170 \mathrm{~K}$, the minimum in $\omega I(\omega)$ is at $\omega \leq 1 \mathrm{GHz}$, so the $\alpha$ peak is completely out of the spectral window. The intensity in the Brillouin frequency region $(\sim 8 \mathrm{GHz})$, which is therefore entirely due to the "fast beta decay", is seen to decrease rapidly with decreasing $T$, consistent with the apparent behavior of $\omega m(\omega)$. This result is also presumably related to the MCT prediction of a cusp in the non-ergodicity parameter $f_{q}(T)$ at $T_{C}$ below which the critical decay weakens with decreasing temperature. It also indicates that additional contributions to the fast relaxation from other processes (e.g. intramolecular vibrations) are unlikely in PC since these would produce a larger value of $B / \Delta^{2}$.

\section{Complete hybrid model fits}

To provide a complete hybrid model analysis, we allowed the ratio $B / \Delta^{2}$ to vary in the fit. At low temperatures, $B$ and $\Delta^{2}$ could be determined independently from the fit. At temperatures above $200 \mathrm{~K}, B$ and $\Delta^{2}$ are strongly correlated. At $210 \mathrm{~K}$ an optimum fit was found with $B / \Delta^{2}=4.0$, and we arbitrarily kept this value fixed for all temperatures above $210 \mathrm{~K}$. The resulting set of fits is shown in Fig. 8; the fitting parameters $B / \Delta^{2}$ and $\tau$ are given in Table II together with the $\chi^{2}$ values. These hybrid model fits have three free parameters, as in the C.D. fits. However, they have $\tau(T)$ constrained to follow Eq. (1) shown by the broken line in Fig. 1, automatically eliminating the $\tau(T)$ disagreement problem of the C.D. fits. They are nevertheless at least as good as the C.D. fits.

In their light scattering study of meta-toluidine, Aouadi et al [Pick 2000] also analyzed $I_{I S O}(\omega)$ spectra with the hybrid model, constraining $\tau_{\alpha}^{C D}$ to be proportional to the $\tau_{\alpha}$ determined from depolarized backscattering spectra. They found, as we did, that at low temperatures the strength of the critical decay decreases with decreasing $T$ (see their Fig. 12). Although they also found that the critical decay strength decreases at high temperatures, resulting in an apparent maximum around $250 \mathrm{~K}$, this behavior may be a manifestation of the strong correlation between $\Delta^{2}$ and $B$ mentioned above.

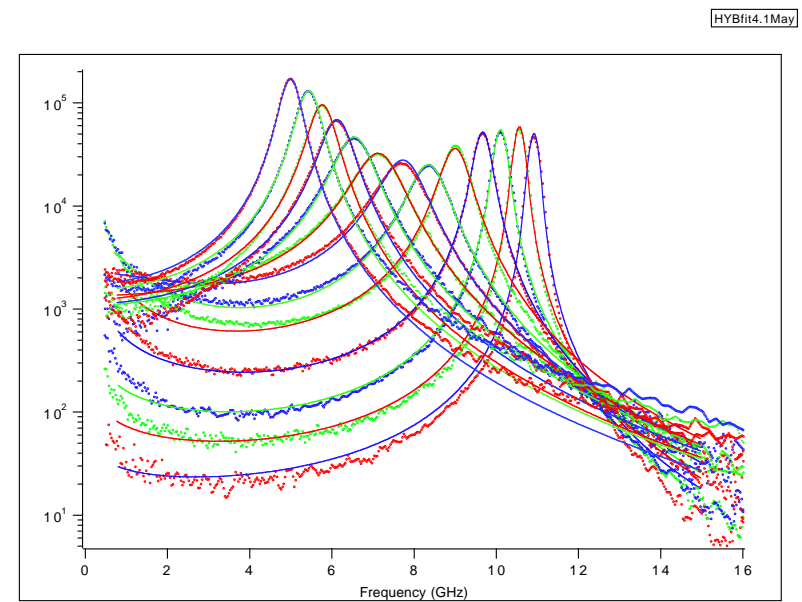

FIG. 8. (HYBFIT4). Fits to PC Brillouin spectra $I_{I S O}(\omega)$ with the hybrid model for $m(\omega)$ of Eq. (7). The free fitting parameters are $I_{0}, \Delta^{2}$, and $B / \Delta^{2}$; the values found from the fits are given in Table II. For $T>210 \mathrm{~K}, \Delta^{2}$ and $B / \Delta^{2}$ were too strongly correlated to be determined by the fit, so $B / \Delta^{2}$ was arbitrarily fixed at 4.0.

\section{E. Thermal diffusion contribution}

With $a_{\text {eff }}$ replacing $a$ in Eq. (7), we carried out additional fits to the Brillouin spectra for $T=150,160$, 170 , and $180 \mathrm{~K}$. The fits are shown by the lower curves for each temperature in Fig. 9. (They are also the four low- $T$ fits shown in Fig. 8 ). Note that at low temperatures the experimental spectra have weak extra structure at low frequencies which is not present in the theoretical fits.

The light scattering spectrum of density fluctuations $I_{I S O}(\omega)$, including thermal diffusion effects, can be expressed as [Boon 1980], [Scopigno 2000]

$$
I(\omega)=\frac{I_{0}}{\omega} \mathcal{I} m\left[\omega_{T}^{2}-\omega^{2}-i \omega \gamma_{0}-\omega m(\omega)\right]^{-1}
$$

where $\omega_{T}=C_{T} q\left(C_{T}\right.$ is the isothermal sound velocity) and $m(\omega)=m_{\eta}(\omega)+m_{T h}(\omega)$ includes both the structural relaxation contribution $m_{\eta}(\omega)$ [e.g. Eqs. (4) or (7)] and the thermal diffusion contribution

$$
m_{T h}(t)=(\gamma-1) \omega_{T}^{2} e^{-t / \tau_{T h}}
$$

where $\tau_{T h}=\left(D_{T} q^{2}\right)^{-1}$. With the Laplace transform convention of Eq. (18) in the Appendix,

$$
m_{T h}(\omega)=\frac{i(\gamma-1) \omega_{0}^{2} \tau_{T h}}{1-i \omega \tau_{T h}}
$$

In the spectral window explored by $90^{\circ}$ Brillouin scattering $(\omega \gtrsim 0.5 \mathrm{GHz}), \omega \tau_{T h}>>1$ since typically $\tau_{T h} \sim$ 
15 ns. Then, in Eq. (10), $-\omega m_{T h}(\omega) \sim \omega_{T}^{2}(\gamma-1)$. Adding this to the $\omega_{T}^{2}$ term in Eq. (10) then gives $\omega_{T}^{2}+\omega_{T}^{2}(\gamma-1)=\omega_{T}^{2} \gamma$ which is just the square of the adiabatic sound frequency $\omega_{0}$. In that approximation Eq. (10) recovers the simpler equation [Eq. (3)] with $m(\omega) \equiv m_{\eta}(\omega)$ only.

While the quasielastic thermal diffusion mode is not usually included in the analysis of Brillouin scattering spectra, it is included in the analysis of inelastic x-ray scattering experiments since with larger $q$ it becomes much broader [Scopigno 2000]. Also, at low temperatures, the high-frequency tail of the thermal diffusion mode can appear in the Brillouin spectrum if the window extends to sufficiently low frequencies. The four spectra shown in Fig. 9 have weak extra structure at low frequencies which may be due to the tail of the thermal diffusion mode.

Since numerical data for $\gamma$ and $D_{T}$ are not available for PC (to our knowledge), we estimated $\gamma=1.5$ and $\tau_{T h}=16 \mathrm{~ns}$, and reanalyzed the spectra of Fig. 9 using Eq. (10) with $\omega_{T}^{2}=\gamma \omega_{0}^{2}, m(\omega)=m_{\eta}(\omega)+m_{T h}(\omega)$ with $m_{T h}(\omega)$ given by Eq. (11), and $m_{\eta}(\omega)$ by Eq. (7). The resulting fits, including thermal diffusion, are shown as the upper curves for each temperature in Fig. 9 for $T=150$, 160 , and $170 \mathrm{~K}$, showing that at these low temperatures, the thermal diffusion mode is apparently visible in the Brillouin spectrum at low frequencies. For temperatures above $170 \mathrm{~K}$, the thermal diffusion contribution is not visible.

\section{F. Hybrid model with $\beta=b$}

Finally, we note that a slightly different strategy can be followed in carrying out the hybrid model analysis. With the exponent $a$ in Eqs. (6) and (7) fixed (for $T>T_{C}$ ) at 0.29 , the region of the minimum in $\omega m^{\prime \prime}(\omega)$ is predicted by MCT to obey Eq. (IV.5) with $b=0.50$. However, there is a slight difference between this prediction and Eq. (7) because so far we have taken $\beta \neq b$. This difference can be eliminated by arbitrarily fixing $\beta=b=0.50$, as was done for the analysis of depolarized backscattering spectra of toulene by Wiedersich et al [Wiedersich 2000].

We therefore carried out another set of hybrid model fits with $\beta=b=0.5$. All other parameters were the same as in the fits of Fig. 8. In these fits, we kept $a=0.29$ and $\beta=0.50$ for all temperatures and also kept both $\Delta^{2}$ and $B$ free. The resulting fits are comparable to those obtained with $\beta=0.68$, demonstrating that the hybrid model fits are not very sensitive to the value of $\beta$.

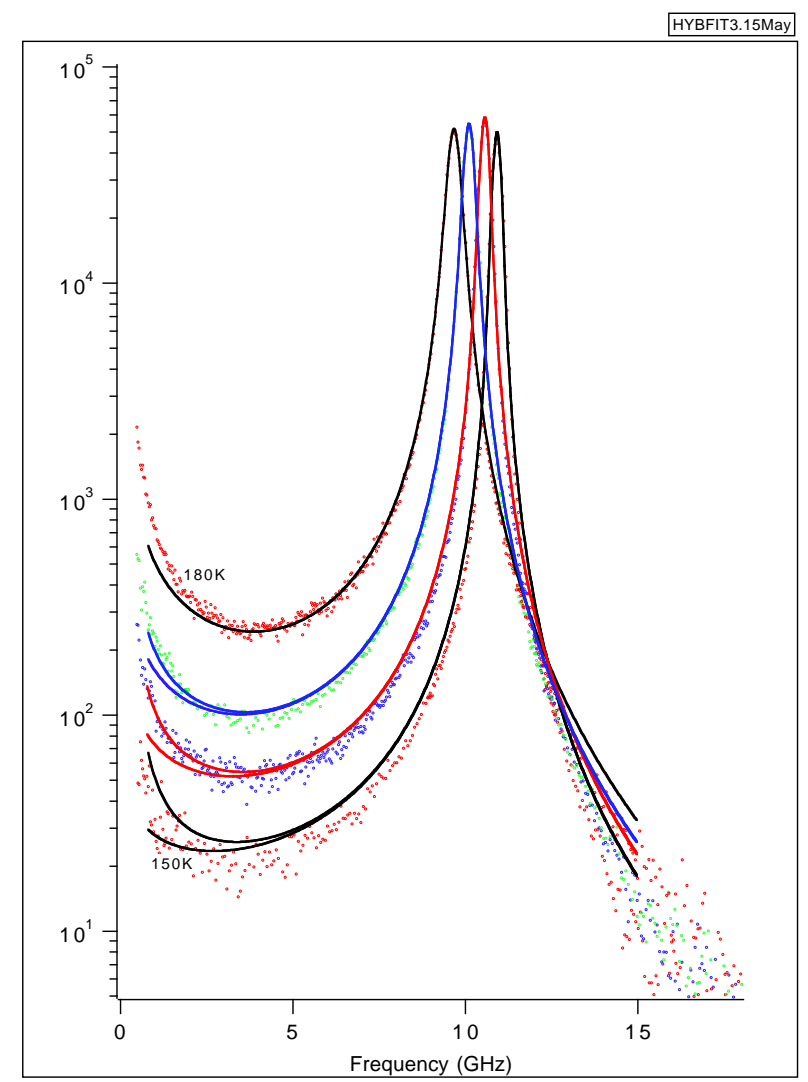

FIG. 9. (HYBFIT3). Low-temperature hybrid model fits using the $a_{\text {eff }}$ values found in the fits shown in Fig. 7. For $T=150,160$, and $170 \mathrm{~K}$, a second fit (the upper curve at each temperature) is included for each spectrum which includes the effect of thermal diffusion.

At low temperatures, however, the low-frequency regions of these fits are not as good as the previous fits, illustrating the effect of the increase in $a_{\text {eff }}$ at low $T$. The $\chi^{2}$ values obtained both with $\beta=0.68$ and $\beta=0.5$ are listed in Table II together with the $B / \Delta^{2}$ values, and are seen to be similar. Note that in the $\beta=0.5$ fits where $\Delta^{2}$ and $B$ were both free fitting parameters, $B / \Delta^{2}$ increases with increasing $T$, passes through a maximum at $T \sim 230 \mathrm{~K}$, and then decreases again at high $T$, similar to the results of Aouadi et al for meta-toluidine [Pick 2000]. As noted above, however, $B$ and $\Delta^{2}$ are strongly correlated at high temperatures, and the high- $T$ values of $B / \Delta^{2}$ are therefore not significant.

An advantage of choosing $\beta=b$ is that there is one less free parameter in the fitting procedure. Also, the region of the susceptibility minimum in Eq. (9) is the same as in Eq. (7) if $\beta=b$. This makes the $\beta=b$ hybrid model a good candidate for attempting to simultaneously describe all relaxing properties in a given material. 


\section{DATA ANALYSIS 3: EXTENDED SCHEMATIC MCT MODEL}

For the third and final analysis of the Brillouin spectra $I_{I S O}(\omega)$, we constructed the memory function $m(\omega)$ of Eq. (3) from the mode coupling theory rather than from parameterized empirical models, using the extended schematic MCT model of Götze and Voigtmann mentioned in the Introduction [Götze 2000].

\section{A. Mode coupling theory}

Mode coupling theory (MCT) begins with exact classical equations of motion (the generalized Langevin equation) for $\phi_{q}(t)$, the normalized autocorrelation functions of the density fluctuations $\rho_{q}(t)$, derived from the Hamiltonian with the Zwanzig-Mori projection-operator formalism [Gotze 1992]:

$\ddot{\phi}_{q}(t)+\nu \dot{\phi}_{q}(t)+\Omega_{q}^{2} \phi_{q}(t)+\Omega_{q}^{2} \int_{0}^{t} m_{q}\left(t-t^{\prime}\right) \dot{\phi}_{q}\left(t^{\prime}\right) d t^{\prime}=0$

Equation (12) is equivalent to the generalized hydrodynamics equation (3), as shown in the Appendix, but its parameters are determined by the Hamiltonian in the Zwanzig-Mori formalism. In particular, $m_{q}\left(t-t^{\prime}\right)$, which is an empirical memory function in Eq. (3), is the autocorrelation function of the random forces in Eq. (12). A central accomplishment of MCT was the derivation of a closed mode-coupling approximation for $m_{q}(t)$ as

$$
m_{q}(t)=\sum_{q_{1}} V\left(q, q_{1}, q-q_{1}\right) \phi_{q_{1}}(t) \phi_{q-q_{1}}(t)
$$

in which the coupling coefficients $V\left(q, q_{1}, q-q_{1}\right)$ are expressed in terms of the static structure factors $S_{q}, S_{q_{1}}$, and $S_{q-q_{1}}$. If the intermolecular potential is known, Eqs. (12) and (13) can be solved self-consistently to find the density correlation functions and spectra [Franosch 1997b].

In cases where the intermolecular potential is not known, simplified "schematic" MCT models can be used which include only a few correlators, with the remaining coupling constants considered as fitting parameters. The Sjögren model is a two-correlator schematic model. The "system" is represented by the system correlator $\phi(t)$, while the variable being probed experimentally is represented by the probe correlator $\phi_{s}(t)$. The two memory functions are

$$
\begin{gathered}
m(t)=v_{1} \phi(t)+v_{2} \phi^{2}(t) \\
m_{s}(t)=V_{s} \phi(t) \phi_{s}(t)
\end{gathered}
$$

Equation (12), written separately for $\phi(t)$ and $\phi_{s}(t)$ with the memory function of Eqs. (14), can then be solved self-consistently with $v_{1}, v_{2}$, and $V_{s}$ as fitting parameters (see [Singh 1998]).

For temperatures near and below $T_{C}$ of MCT, the "cage effect" dynamics embodied in Eq. (12) lead to the (usually) non-physical result of total structural arrest. In most glass-forming materials, this arrest is avoided by activated hopping processes which are included in the extended version of MCT (to a first approximation) via a temperature-dependent hopping parameter $\Delta$. Equation (12) is replaced by [Götze 2000]

$$
\begin{array}{r}
\ddot{\phi}(t)+(\Delta+\nu) \dot{\phi}(t)+\left(\Omega^{2}+\Delta \cdot \nu\right) \phi(t) \\
+\Omega^{2} \int_{0}^{t} m\left(t-t^{\prime}\right)\left[\dot{\phi}\left(t^{\prime}\right)+\Delta \cdot \phi\left(t^{\prime}\right)\right] d t^{\prime}=0
\end{array}
$$

(Note that the hopping parameter $\Delta$ in Eq. (15) is not related to the coupling constant $\Delta^{2}$ of the Brillouin fits in, e.g., Eq. (7).)

The extended Sjögren model of Götze and Voigtmann consists of two Eqs. (15), one for $\phi$ and one for $\phi_{s}$, with the two memory functions of Eqs. (14). These are to be solved self-consistently.

The parameters of $\phi(t)$ are: $\Omega, \nu, \Delta, v_{1}$, and $v_{2}$. They have already been globally optimized for PC by Götze and Voigtmann for dielectric, neutron scattering and depolarized light scattering data covering a much larger range of frequencies than that of our Brillouin scattering spectra. The parameters of $\phi_{s}(t)$ are: $\Omega_{s}, \nu_{s}, \Delta_{s}$, and $V_{s}$. (Note that varying the parameters of $\phi_{s}$ has no effect on $\phi$.)

In this section, the Götze-Voigtmann schematic MCT model will be used to compute the memory function $m(\omega)$ of Eq. (3), taken as $m(\omega)=\Delta^{2} \phi_{s}(\omega)$, where $\Delta^{2}$ is the coupling constant. [We note that this is the same procedure employed by Rufflé et al [Rufflé 1999] who used the simpler Sjögren model)].

\section{B. MCT fitting procedure}

Fitting the Brillouin spectra $I_{I S O}(\omega)$ with the twocorrelator MCT model described above is technically more difficult and therefore less straightforward than the two other fitting procedures. This is because $m(\omega)=$ $\phi_{s}(\omega)$ must first be obtained by solving and Fourier transforming the MCT equations [Eqs. (15)], and then interpolated to match the frequencies of the $I_{I S O}(\omega)$ spectrum.

For the fits with the schematic MCT model, we began with the parameters obtained in the depolarized light scattering (DLS) fits of Götze and Voigtmann, shown in their Figure 1 [Götze 2000]. Since the correlators $\phi_{s}$ computed with the parameters they used gave very good fits to the depolarized light scattering spectra over nearly four decades in frequency, we tried to fit the Brillouin 
scattering spectra using these same parameters. However, the $\alpha$ peaks of the DLS spectra are at lower frequencies than the corresponding $\tau_{\alpha}^{L A}$ values, as seen in Fig. 1, where $\tau_{\alpha}^{D L S} \sim 5 \times \tau_{\alpha}^{L A}$. We found that the position of the $\alpha$ peak in $\omega \phi_{s}^{\prime \prime}(\omega)$ is primarily determined by the coupling constant $V_{s}$ in Eq. (14b). We therefore treated $V_{s}$ as a free fitting parameter. All other MCT parameters were kept at their DLS values. We solved the MCT equations [Eqs. (15)] for selected values of $V_{s}$ and the resulting $\phi_{s}(\omega)$, after multiplication by the (adjustable) coupling constant $\Delta^{2}$, was taken as the memory function $m(\omega)$ in Eq. (3). A nonlinear least-squares analysis was then carried out in which $I_{0}$ and $\Delta^{2}$ were the free parameters. This process was repeated until the fit was optimized. (Note that in fitting their Brillouin spectra of $\mathrm{NaLiPO}_{3}$ with the idealized two-correlator schematic Sjögren model, Rufflé et al also treated $V_{s}$ as a free parameter while the system parameters were fixed from neutron scattering results [Rufflé et al 1999].)

The MCT fits for $T=160,180,200,220,240,260$, 280, 300, 320, and $350 \mathrm{~K}$ are shown in Fig. 10. (For $T \geq 300 \mathrm{~K}$, the MCT parameters other than $V_{s}$ were obtained by extrapolation from lower temperatures.) The values $V_{s}$ and $\chi^{2}$ for these fits are given in Table II (note that the $V_{s}$ values for the Brillouin fits are systematically smaller than the depolarized light scattering values $V_{s}^{D L S}$ ). The MCT fits are comparable to the hybrid fits, and have lower $\chi^{2}$ values at some temperatures. For the lowest temperatures, the low-frequency region of the $\mathrm{MCT}$ fits is poor, reflecting the known limitations of the model for low frequencies at $T<T_{C}$ [Götze 2000b]. At temperatures above $280 \mathrm{~K}$, the fits were not sensitive to the value of $V_{s}$ for $V_{s} \lesssim 3.5$. This is because the $\alpha$ peak in $\omega m^{\prime \prime}(\omega)$ is then above the Brillouin line, and the shape of the memory function in the Brillouin range (1 to $15 \mathrm{GHz}$ ) is nearly independent of $V_{s}$.

For the temperatures 180, 200, 220, 240, and $260 \mathrm{~K}$, the position of the $\alpha$ peak in $\omega m^{\prime \prime}(\omega)$ was sufficiently welldetermined by the fits to estimate the MCT $\alpha$-relaxation time $\tau_{\alpha}=1 / \omega_{\alpha}$ from the frequency of the $\alpha$ peak. The values found are listed in Table II and are also shown in Fig. 1 by the $*$ symbols. Note that the MCT values follow the same qualitative temperature dependence as the dielectric data, and are similar to (though somewhat lower than) the values we assumed for the hybrid fits. Thus, the MCT approach, in contrast to the other approaches followed in the preceding two sections, is able to provide reasonable estimates of $\tau_{\alpha}^{L A}$ purely on the basis of fits to the Brillouin spectra.

These results show that this extended schematic MCT model is able to simultaneously describe the spectra and also give reasonable fits for $\tau_{\alpha}(T)$, thus answering the question that originally motivated this study affirmatively.

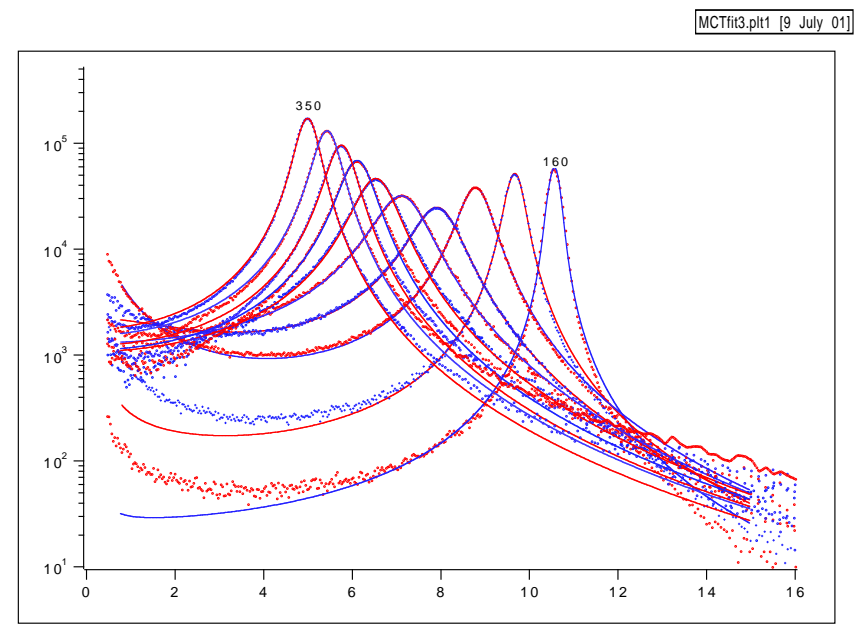

FIG. 10. (MCTFIT3). Fits of PC $I_{I S O}(\omega)$ spectra with the extended schematic MCT model of Eqs. (15) and (14) for $T=350,320,300,280,260,240,220,200,180$, and $160 \mathrm{~K}$. In the fits, $\gamma_{0}(T)=T / 1000$ and $\omega_{0}(T)$ is fixed following Eq. (5). All MCT parameters are those found by Götze and Voigtmann from their fits to DLS spectra except for $V_{s}$ which has been adjusted to optimize the fits. For $T \geq 300 \mathrm{~K}$, the parameters other than $V_{s}$ were determined by extrapolation from lower temperatures.

\section{DISCUSSION AND CONCLUSIONS}

As noted over 30 years ago by Montrose et al, the detailed shape of the $I_{I S O}(\omega)$ Brilliouin spectrum is relatively insensitive to the exact nature of the memory function [Montrose 1968]. This is due, as noted earlier, to the limited frequency range of Brillouin scattering data. If $\omega_{0}$ is fixed (from ultrasonic data), the ratio $m^{\prime \prime}\left(\omega_{B}\right) / m^{\prime}\left(\omega_{B}\right)$ must have the "correct" value to obtain reasonable fits, but there is still enormous flexibility in constructing $m(\omega)$. If one wishes to extract meaningful information from the fits, then additional constraints must be imposed.

First, the $\alpha$ relaxation time should be reasonable. Fits using $\alpha$-relaxation-only models, e.g. the Cole-Davidson function of Eq. (4), produce acceptable fits but give unreasonably short relaxation times at low temperatures. At $T \sim T_{G}$, one finds typically $\tau_{\alpha}^{C D}\left(T_{G}\right) \sim 10^{-7} \mathrm{sec}$ while other experimental techniques generally yield $\tau_{\alpha}\left(T_{G}\right) \sim 10^{2}$ sec. This result demonstrates the fact, already shown many times, that $\alpha$-only models of structural relaxation are incomplete.

A great deal of research during the past 15 years has shown that structural relaxation occurs in two steps, starting with a fast decay towards a plateau that precedes 
the final $\alpha$-relaxation process. Since the memory function $m(\omega)$ for longitudinal viscosity should also have this two-step form, the memory function used in the analysis of Brillouin scattering spectra should include both the $\alpha$ relaxation and the fast-relaxation parts of $m(\omega)$. If $\tau_{\alpha}$ is constrained to follow the temperature dependence found in other measurements, such as dielectric or depolarized light scattering spectroscopy, then the memory function must be extended to higher frequencies to provide sufficient damping once the $\alpha$ peak in $\omega m^{\prime \prime}(\omega)$ has moved below the Brillouin lines. This extension to include fast relaxation processes can be implemented many ways, but most approaches require the introduction of a fast $\beta$ relaxation time $\tau_{\beta}$ that does not correspond to the results of other experiments.

In practice, there may also be other sources of fast relaxation - such as intramolecular vibrations - that influence the Brillouin spectra, but the high-frequency part of the structural relaxation dynamics must still be present.

Because the mode coupling theory has been successful in analyzing a wide range of experimental data, we have used an empirical model for $m(\omega)$ which is qualitatively consistent with MCT. The hybrid model, which mimics the two-step relaxation scenario of MCT by combining the C.D. function for $\alpha$ relaxation with the critical decay law $\left(\omega^{a}\right)$ has been shown to provide generally excellent fits to the Brillouin scattering spectra.

Furthermore, we found that fixing $\beta=b=0.5$ produces fits of equally good quality as with $\beta=0.68$. This reduction in the number of free fitting parameters represents an attractive variant of the hybrid model which has been shown, for the case of toluene, to provide good fits to the depolarized backscattering spectra [Wiedersich 2000]. It will be interesting to explore the ability of this reduced hybrid model to simultaneously fit both depolarized backscattering spectra and Brillouin spectra for any given material.

We have also explored the applicability of the twocorrelator schematic MCT model to the analysis of Brillouin spectra, a procedure first employed by Rufflé et al [Rufflé 1999] who used the Sjögren model without hopping. We used the new extended model of Götze and Voigtmann [Götze 2000] with which they fit depolarized light scattering, neutron scattering, and dielectric data for propylene carbonate with common parameters for the system correlator $\phi(t)$ for all the experiments. For the probe correlator $\phi_{s}(t)$, we kept the parameters $\Omega_{s}$, $\nu_{s}$, and $\Delta_{s}$ they obtained from fits to depolarized lightscattering spectra fixed, but we varied $V_{s}$ to optimize the fits to the Brillouin spectra, taking $m(\omega)=\Delta^{2} \phi_{s}(\omega)$ where $\Delta^{2}$ is a free parameter. The resulting $V_{s}$ values were systematically smaller [and the resulting frequency of the $\alpha$ peak in $\omega m^{\prime \prime}(\omega)$ higher] than those found from fits to the depolarized light scattering spectra, consistent with the result that $\tau_{\alpha}^{L A}$ is smaller than $\tau_{\alpha}$ of depolarized light-scattering spectra as shown in Fig. 1. We then used the positions of the $\alpha$ peaks in the MCT memory function to estimate the relaxation time $\tau_{\alpha}^{M C T}=1 / \omega_{\alpha}^{M C T}$. As seen in Fig. 1, the resulting $\tau_{\alpha}^{M C T}$ values follow the temperature dependence expected on the basis of the dielectric data of Schneider et al. We emphasize that this MCT analysis is the only one of the three employed in this study that can provide a meaningful estimate of $\tau_{\alpha}$.

The recent Brillouin scattering study of OTP by Monaco et al [Monaco 1999, Monaco 2000, Monaco 2001, Mossa 2001] has suggested a different origin for the fast relaxation process observed in Brillouin scattering spectra: coupling of longitudinal acoustic waves to intramolecular degrees of freedom as originally suggested by Mountain [Mountain 1968]. Even in OTP, however, where this mechanism appears to be important, some part of the fast relaxation must be associated with structural relaxation. It is therefore an open question, that should be carefully addressed, how much of the fast relaxation originates in each of these two mechanisms, in OTP as well as in other materials - including PC.

Finally, we return to the question of the ability of Brillouin scattering spectra to extract meaningful information about the structural relaxation process. In Fig. 11 we show $\omega m^{\prime \prime}(\omega)$ (solid lines) and $\omega m^{\prime}(\omega)$ (broken lines) obtained from the MCT fits (top) and the hybrid fits with $\beta=b$ (bottom).
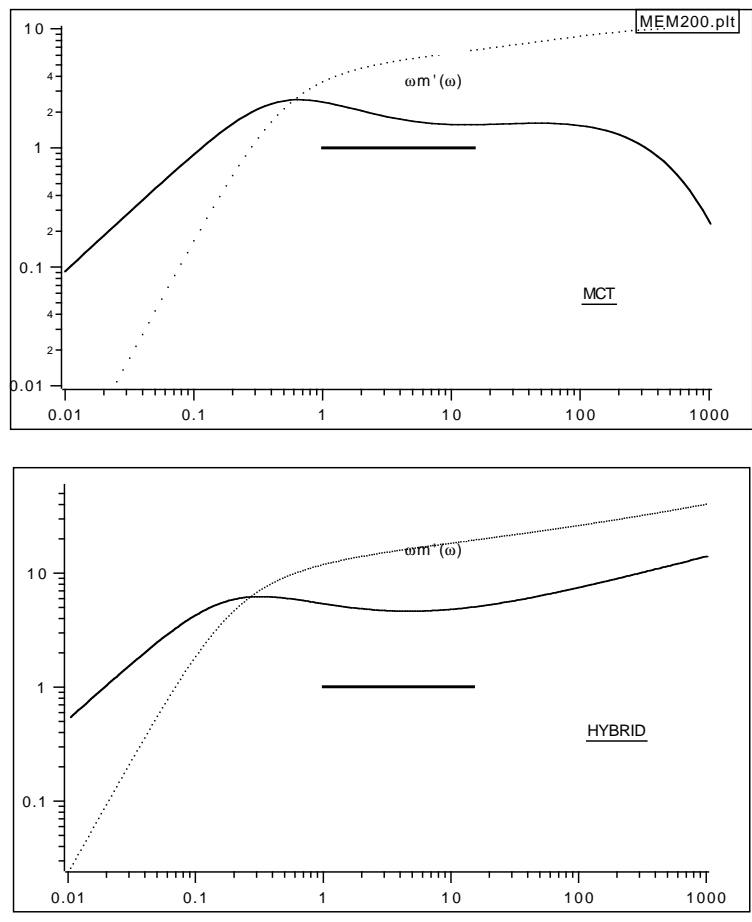

FIG. 11. (MEMPLOT200). $\omega m^{\prime \prime}(\omega)$ (solid lines) and $\omega m^{\prime}(\omega)$ (broken lines) from the fits at $200 \mathrm{~K}$. Top: MCT fit; bottom: hybrid fit with $\beta=b=0.5$ and $a=0.29$. The fit range $(1-15 \mathrm{GHz})$ is indicated in both plots by a thick horizontal line. 
In the range of the Brillouin spectra $(1-15 \mathrm{GHz}$ indicated by the horizontal solid lines), the shapes of these curves are remarkably close, even though they were obtained with very different procedures. This result suggests that a reasonable phenomenological model like the hybrid model, with the constraint that $\tau_{\alpha}$ should have a temperature dependence consistent with that found by other techniques, can provide a credible representation of the memory function $m(\omega)$ characterizing the longitudinal viscosity.

\section{ACKNOWLEDGMENTS}

We thank W. Götze, J. Wiedersich, M. Fuchs, and R. Pick for helpful discussions and suggestions, Th. Voigtmann for providing the computer programs (and extensive advice) for the schematic MCT analysis, and P. Lunkenheimer for providing the $\tau_{\alpha}(T)$ values shown in Fig. 1. HZC thanks the Alexander von Humboldt Foundation for support, and the Technical University of Munich for hospitality during a visit when this project was begun.

This research was supported by the National Science Foundation under Grants DMR-9616577 and DMR9980370. Travel support for the CCNY-TUM collaboration was provided by NATO under Collaborative Research Grant No. CRG-930730.

\section{APPENDIX: SOME MATHEMATICAL DETAILS}

The normalized density fluctuation correlation function

$$
\phi(t)=<\rho_{q}(0) \rho_{q}(t)>/<\left|\rho_{q}(0)\right|^{2}>
$$

obeys the generalized Langevin (or generalized oscillator) equation

$$
\ddot{\phi}(t)+\gamma_{0} \dot{\phi}(t)+\omega_{0}^{2} \phi(t)+\int_{0}^{t} m\left(t-t^{\prime}\right) \dot{\phi}\left(t^{\prime}\right) d t^{\prime}=0
$$

$$
\begin{aligned}
\omega m(\omega) & =\Delta^{2}\left[(1-i \omega \tau)^{-\beta}-1\right]+i \omega B \times \Gamma(1-a)\left[\tau^{-1}-i \omega\right]^{a-1} \\
& =\Delta^{2}\left[(1-i \omega \tau)^{-\beta}-1\right]+i \omega B \times \Gamma(1-a) \tau^{1-a}(1-i \omega \tau)^{a-1}
\end{aligned}
$$

which is Eq. (7) in the text. (For $a=0.29, \Gamma(1-a)=$ 1.282.) 


\section{REFERENCES}

* present address: Physics Department, Emory University, Atlanta, GA 30322.

[1] [Boon 1980] J.P. Boon and S. Yip, "Molecular Hydrodynamics", McGraw-Hill, 1980 (reprinted by Dover Publications).

[2] [Chung 1971] C.-H. Chung and S. Yip, Phys. Rev. A 4, 928 (1971).

[3] [Cummins 1994] H.Z. Cummins, G. Li, W.M. Du, and J. Hernandez, J. Non-Cryst. Solids 172-174, 26 (1994).

[4] [Cummins 1998] H.Z. Cummins, Y.H. Hwang, Gen Li, W.M. Du, W. Losert, and G.Q. Shen, J. Non-Cryst. Solids 235-237, 254 (1998).

[5] [Dreyfus 1999] C. Dreyfus, A. Aouadi, R.M. Pick, T. Berger, A. Patkowski, and W. Steffen, Eur. Phys. J. B9, 401 (1999).

[6] [Du 1994] W.M. Du, G. Li, H.Z. Cummins, M. Fuchs, J. Toulouse, and L.A. Knauss, Phys. Rev. E 49, 2192 (1994).

[7] [Du 1996] W.M. Du, Ph.D. dissertation, the City University of New York, 1996.

[8] [Elmroth 1992] M. Elmroth, L. Borjesson, and L.M. Torell, Phys. Rev. Lett. 68, 79 (1992).

[9] [Fioretto 1999] D. Fioreto, L. Comez, G. Socino, L. Verdini, S. Corezzi, and P.A. Rolla, Phys. Rev. E 59, 1899 (1999); G. Monaco, L. Comez, and D. Fioretto, Phil. Mag. B 77, 463 (1998).

[10] [Frank 2000] M. Frank, Diploma Thesis, The Technical University of Munich (November, 2000).

[11] [Franosch 1997] T. Franosch, W. Götze, M.R. Mayr, and A.P. Singh, Phys. Rev. E 55, 3183 (1997).

[12] [Franosch 1997b] T. Franosch, M. Fuchs, W. Götze, M.R. Mayr, and A.P. Singh, Phys. Rev. E 55, 7153 (1997).

[13] [Gotze 1992] W. Götze and L. Sjögren, Rep. Prog. Phys. 55, 241 (1992).

[14] [Gotze 1993] W. Götze, private communication.

[15] [Gotze 2000] W. Götze and Th. Voigtmann, Phys. Rev. E 61, 4133 (2000).

[16] [Gotze 2000b] W. Götze - private communication.

[17] [Gradshteyn 1965] I.S. Gradshteyn and I.M. Ryzhik, in: Table of Integrals, Series, and Products, 4th Ed. (Academic Press, 1965).

[18] [Latz 2000] A. Latz and M. Letz, Eur. Phys. J. B 19, 323 (2001).

[19] [Lebon 1997] M.J. Lebon, C. Dreyfus, Y. Guissani, R.M. Pick, and H.Z. Cummins, Z. Phys. B 103, 433 (1997).

[20] [Loheider 1990] S. Loheider, G. Vogler, I. Petscherizin, M. Soltwisch, and D. Quitmann, J. Chem. Phys. 93, 5436 (1990).

[21] [Monaco 1999] G. Monaco, D. Fioretto, C. Masciovecchio, G. Ruocco, and F. Sette, Phys. Rev. Lett. 82, 1776 (1999).

[22] [Monaco 2000] G. Monaco, S. Caponi, R. DiLeonardo, D. Fioretto, and G. Ruocco, Phys. Rev. E 62, R7595 (2000).
[23] [Monaco 2001] G. Monaco, D. Fioretto, L. Comez, and G. Ruocco, Phys. Rev. E 63, 061502 (2001).

[24] [Montrose 1968] C.J. Montrose, V.A. Solovyev, and T.A. Litovitz, J. Acoust. Soc. Am. 43, 117 (1968).

[25] [Mossa 2001] S. Mossa, G. Monaco, and G. Ruocco, Cond. Matt. /0104265 (2001).

[26] [Mountain 1968] R.D. Mountain, J. Res. NBS 70A, 207 (1966); 72 A, 95 (1968).

[27] [Pick 2000] A. Aouadi, C. Dreyfus, M. Massot, R.M. Pick, T. Berger, W. Steffen, A. Patkowski, and C. AlbaSimionesco, J. Chem. Phys. 112, 9860 (2000).

[28] [Ruffle 1999] B. Rufflé, C. Ecolivet, and B. Toudic, Europhys. Lett. 45, 591 (1999).

[29] [Schneider 1999] U. Schneider, P. Lunkenheimer, R. Brand, and A. Loidl, Phys. Rev. E 59,6924 (1999).

[30] [Scopigno 2000] T. Scopigno, U. Balucani, G. Ruocco, and F. Sette, Phys. Rev. Lett. 85, 4076 (2000).

[31] [Shen 1998] G. Shen, Ph.D. Dissertation, The City University of New York, 1998.

[32] [Singh 1998] A.P. Singh, G. Li, W. Götze, M. Fuchs, T. Franosch, and H.Z. Cummins, J. Non-Cryst. Solids 235237, 66 (1998).

[33] [Sjogren 1986] L. Sjögren, Phys. Rev. A 33, 1254 (1986).

[34] [Soltwisch 1998] M. Soltwisch, G. Ruocco, B. Baushun, J. Bosse, V. Mazzacurati, and D. Quitmann, Phys. Rev. E 57, 720 (1988).

[35] [Wiedersich 2000] J. Wiedersich, N. Surovtsev, and E. Rossler, J. Chem. Phys. 113, 1143 (2000).

[36] [Note IIIB.1] In carrying out fits to Eq. (III.1) with the memory functions $m(\omega)$ of Eqs. (III.2) or (III.9), we used the following conventions for units: $\omega, \omega_{0}, \gamma_{0}$, and $\Delta$ are all in $\mathrm{GHz}$, while $\tau$ is in nsec. In calculating $\omega \tau$, we therefore multiplied $\tau$ by $2 \pi$. 
TABLE I. Properties of Propylene Carbonate

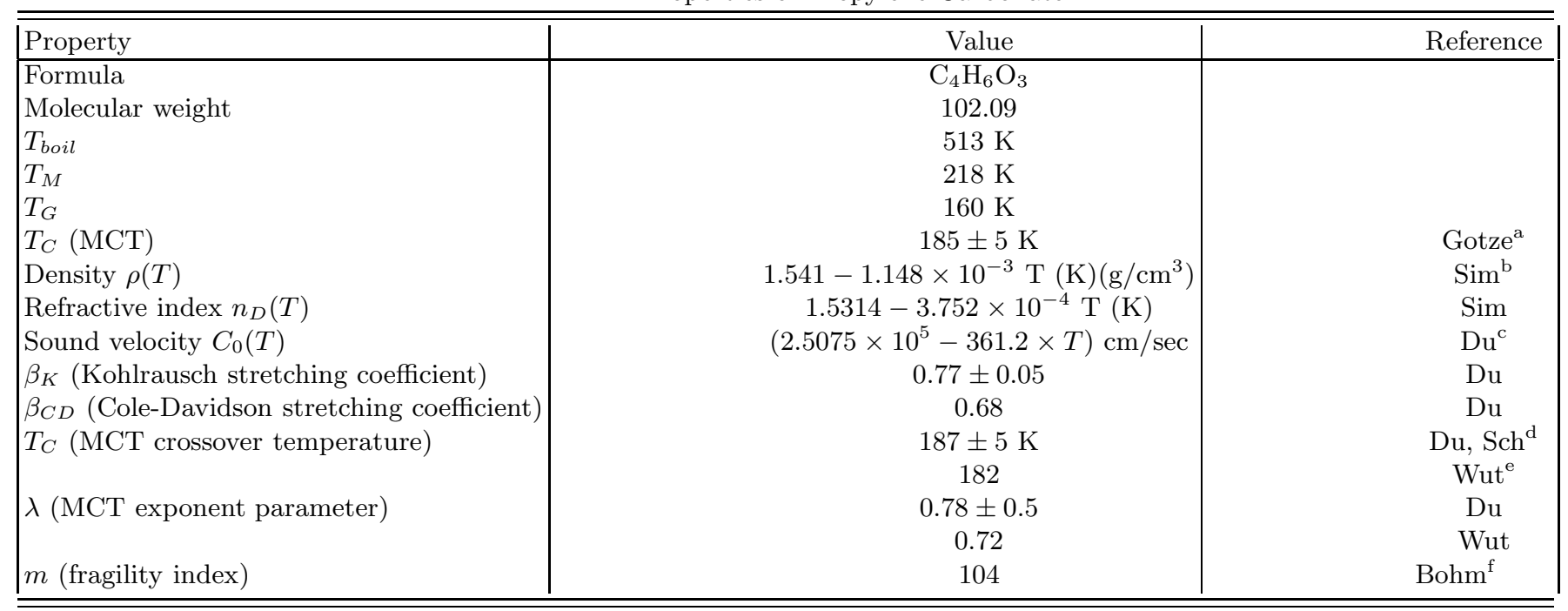

a (Gotze) W. Götze and Th. Volgtmann, Phys. Rev. E 61, 4133 (2000).

b(Sim) L. Simeral and R.L. Amey, J. Phys. Chem. 74, 1443 (1970).

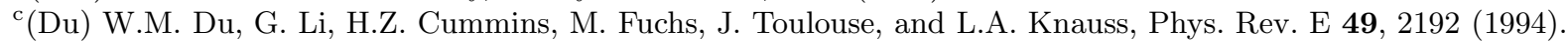

${ }^{\mathrm{d}}$ (Sch) U. Schneider, P. Lunkenheimer, R. Brand, and A. Loidl, Phys. Rev. E 59, 6924 (1999).

e(Wut) J. Wuttke, M. Ohl, M. Goldhammer, S. Roth, U. Schneider, R. Lunkenheimer, R. Kahn, B. Rufflé, R. Lechner, and M.A. Berg (submitted to Phys. Rev. E).

${ }^{\mathrm{f}}$ (Bohm) R. Böhmer, K.L. Ngai, C.A. Angell, and D.J. Plazek, J. Chem. Phys. 99, 4201 (1993).

TABLE II.

\begin{tabular}{|c|c|c|c|c|c|c|c|c|c|c|c|c|}
\hline \multirow{3}{*}{$T(K)$} & \multirow{2}{*}{\multicolumn{3}{|c|}{ C.D. $($ free $\tau)$}} & \multicolumn{5}{|c|}{ Hybrid (fixed $\tau$ ) } & \multicolumn{4}{|c|}{ Schematic MCT } \\
\hline & & & & & $\beta=$ & & $\beta=b$ & $=0.5$ & $\tau(\mathrm{ns})$ & $V_{s}^{D L S}$ & $V_{s}^{B R}$ & $\chi^{2}$ \\
\hline & $\tau(\mathrm{ns})$ & $\Delta^{2}\left(\mathrm{GHz}^{2}\right)$ & $\chi^{2}$ & $\tau(\mathrm{ns})$ & $B / \Delta^{2}$ & $\chi^{2}$ & $B / \Delta^{2}$ & $\chi^{2}$ & & & & \\
\hline 140 & 790 & 36.5 & 27.2 & $1.26 \times 10^{22}$ & & & & & & & & \\
\hline 150 & 1220 & 40.2 & 27.8 & $1.42 \times 10^{14}$ & 0.01 & 27.4 & 0.002 & 28.6 & & 94 & & \\
\hline 160 & 31.8 & 37.0 & 22.1 & $2.49 \times 10^{8}$ & 0.04 & 19.5 & 0.034 & 22.7 & & 80 & 30 & 25.2 \\
\hline 165 & 14.2 & 34.3 & 23.3 & $1.96 \times 10^{\circ}$ & & & & & & & & \\
\hline 185 & 0.763 & 25.3 & 84.6 & $3.40 \times 10^{1}$ & & & & & & & & \\
\hline 195 & 0.236 & 22.0 & 60.4 & 2.51 & 1.50 & 47.3 & & & & & & \\
\hline 200 & 0.148 & 20.6 & 44.2 & $9.75 \times 10^{1}$ & & 35.2 & 1.31 & 23.3 & $2.4 \times 10^{-1}$ & 30 & 7.6 & 5.85 \\
\hline 205 & $1.03 \times 10^{-1}$ & 19.5 & 28.8 & $4.47 \times 10^{-1}$ & & & & & & & & \\
\hline 210 & $7.28 \times 10^{-2}$ & 18.4 & 16.4 & $2.33 \times 10^{-1}$ & 4.00 & 10.1 & 2.72 & 8.3 & & 25 & & \\
\hline 215 & $5.41 \times 10^{-2}$ & 17.6 & 17.8 & $1.34 \times 10^{-1}$ & & & & & & & & \\
\hline 240 & $1.75 \times 10^{-2}$ & 17.0 & 11.9 & $2.16 \times 10^{-2}$ & 4.00 & 14.2 & 24.8 & 13.2 & $9.9 \times 10^{-3}$ & 18 & 4.7 & 11.9 \\
\hline 250 & $1.28 \times 10^{-2}$ & 17.0 & 25.7 & $1.36 \times 10^{-2}$ & & & & & & 16 & & \\
\hline 260 & $9.96 \times 10^{-3}$ & 17.0 & 50.0 & $9.29 \times 10^{-3}$ & 4.00 & 41.6 & 21.2 & 41.3 & $4.7 \times 10^{-3}$ & 15 & 4.3 & 39.9 \\
\hline 270 & $8.17 \times 10^{-3}$ & 17.0 & 47.4 & $6.80 \times 10^{-3}$ & & & & & & 13 & & \\
\hline 280 & $6.61 \times 10^{-3}$ & 17.0 & 66.3 & $5.24 \times 10^{-3}$ & 4.00 & 54.3 & 22.0 & 54.0 & & 12 & $<3.5$ & 51.9 \\
\hline 290 & $5.58 \times 10^{-3}$ & 17.0 & 60.5 & $4.19 \times 10^{-3}$ & & & & & & & & \\
\hline 300 & $4.57 \times 10^{-3}$ & 17.0 & 60.7 & $3.46 \times 10^{-3}$ & 4.00 & 53.5 & 0.87 & 53.5 & & & $<2.5$ & \\
\hline 310 & $3.90 \times 10^{-3}$ & 17.0 & 43.4 & $2.93 \times 10^{-3}$ & & & & & & & & \\
\hline 320 & $3.38 \times 10^{-3}$ & 17.0 & 49.2 & $2.53 \times 10^{-3}$ & 4.00 & 44.3 & 2.34 & 44.3 & & & $<2.5$ & 40.5 \\
\hline 330 & $2.76 \times 10^{-3}$ & 17.0 & 38.1 & $2.22 \times 10^{-3}$ & & & & & & & & \\
\hline 340 & $2.26 \times 10^{-3}$ & 17.0 & 26.3 & $1.98 \times 10^{-3}$ & & & & & & & & \\
\hline
\end{tabular}

